\title{
FINITE ELEMENTS FOR SYMMETRIC TENSORS IN THREE DIMENSIONS
}

\author{
DOUGLAS N. ARNOLD, GERARD AWANOU, AND RAGNAR WINTHER
}

\begin{abstract}
We construct finite element subspaces of the space of symmetric tensors with square-integrable divergence on a three-dimensional domain. These spaces can be used to approximate the stress field in the classical Hellinger-Reissner mixed formulation of the elasticty equations, when standard discontinuous finite element spaces are used to approximate the displacement field. These finite element spaces are defined with respect to an arbitrary simplicial triangulation of the domain, and there is one for each positive value of the polynomial degree used for the displacements. For each degree, these provide a stable finite element discretization. The construction of the spaces is closely tied to discretizations of the elasticity complex and can be viewed as the three-dimensional analogue of the triangular element family for plane elasticity previously proposed by Arnold and Winther.
\end{abstract}

\section{INTRODUCTION}

The classical Lagrange finite element spaces provide natural simplicial finite element discretizations of the Sobolev space $H^{1}$. Similarly, various finite element spaces derived in the theory of mixed finite elements, such as the Raviart-Thomas and Nedelec spaces, provide the natural finite element discretizations of the spaces $H$ (div) and $H$ (curl). (These statements are made precise and treated in a uniform framework of the finite element exterior calculus in [9].) In this paper we consider the finite element discretization of the space $H(\operatorname{div}, \Omega ; \mathbb{S})$ consisting of square-integrable symmetric tensors (or, given a choice of coordinates, symmetric matrix fields) with square-integrable divergence. In the classical Hellinger-Reissner mixed formulation of the elasticity equations, the stress is sought in $H(\operatorname{div}, \Omega ; \mathbb{S})$ and the displacement in $L^{2}\left(\Omega ; \mathbb{R}^{n}\right)$. The natural discretization of the latter space is evident-piecewise polynomial of some degree without interelement continuity constraints - but the development of an appropriate finite element subspace of $H(\operatorname{div}, \Omega ; \mathbb{S})$ to use with these is a long-standing and challenging problem. For plane elasticity, the known stable mixed finite element methods have mostly involved composite elements for the stress [6, 15, 16, 21. To avoid these, other authors have modified the standard mixed variational formulation of elasticity to a formulation that uses general, rather than symmetric, tensors for the stress, with the symmetry imposed weakly; see [2, 5, 7, 17, 18, 19, 20, 8. Not until 2002 was a stable non-composite finite method for the classical mixed formulation of plane elasticity found [10]. This work can be seen as answering the question "what are

Received by the editor January 17, 2007 and, in revised form, May 8, 2007 and December 4, 2007.

2000 Mathematics Subject Classification. Primary 65N30; Secondary 74S05.

(C)2008 American Mathematical Society 1229

Reverts to public domain 28 years from publication 
the natural finite element discretizations of $H(\operatorname{div}, \Omega ; \mathbb{S})$ ?" in the case of two dimensions. In this paper we address this question for three dimensions.

Up until recently, there were no mixed finite elements for the Hellinger-Reissner formulation in three dimensions known to be stable. In [1], a partial analogue of the lowest order element in [10] was proposed and shown to be stable. Here we will derive the full analogue of the results of [10. We construct a family of finite element subspaces of $H(\operatorname{div}, \Omega ; \mathbb{S})$ that, when used to discretize the stress in elasticity along with the obvious discontinuous piecewise polynomial discretization for the displacement, provide stable mixed finite elements for the Hellinger-Reissner principle. As in two dimensions, these spaces are related to a finite element subcomplex of the elasticity complex, related to it by commuting diagrams.

We recall the standard mixed formulation for the elasticity equations. Let $\Omega$ be a contractible polyhedral domain in $\mathbb{R}^{3}$, occupied by a linearly elastic body which is clamped on the boundary $\partial \Omega$, and let $S$ and $u$ denote the stress and displacement fields engendered by a force $f$ acting on the body. The matrix field $S$ and the vector field $u$ can be characterized as the unique critical point of the Hellinger-Reissner functional

$$
\mathcal{J}(T, v)=\int_{\Omega}\left(\frac{1}{2} A T: T+\operatorname{div} T \cdot v-f \cdot v\right) d x
$$

over the space $H(\operatorname{div}, \Omega ; \mathbb{S}) \times L^{2}\left(\Omega ; \mathbb{R}^{3}\right)$. Here, $\mathbb{S}$ is the six-dimensional space of symmetric matrices and $S: T$ denotes the Frobenius product on $\mathbb{S}$. The given compliance tensor $A=A(x): \mathbb{S} \rightarrow \mathbb{S}$ is symmetric, and bounded and positive definite uniformly with respect to $x \in \Omega$. The divergence operator, div, is applied to a matrix field by taking the divergence of each row. Hence, this operator maps the space $H(\operatorname{div}, \Omega ; \mathbb{S})$ into $L^{2}\left(\Omega ; \mathbb{R}^{3}\right)$.

A mixed finite element method determines an approximate stress field $S_{h}$ and an approximate displacement field $u_{h}$ as the unique critical point $\left(S_{h}, u_{h}\right)$ of the Hellinger-Reissner functional in a finite element space $\Sigma_{h} \times V_{h} \subset H(\operatorname{div}, \Omega ; \mathbb{S}) \times$ $L^{2}\left(\Omega ; \mathbb{R}^{3}\right)$, where $h$ denotes the mesh size. Equivalently, $\left(S_{h}, u_{h}\right) \in \Sigma_{h} \times V_{h}$ solves the saddle point system

$$
\int_{\Omega}\left(A S_{h}: T+\operatorname{div} T \cdot u_{h}+\operatorname{div} S_{h} \cdot v\right) d x=\int_{\Omega} f v d x, \quad(T, v) \in \Sigma_{h} \times V_{h} .
$$

To ensure that the discrete system has a unique solution and that it provides a good approximation of the true solution, the finite-dimensional spaces $\Sigma_{h}$ and $V_{h}$ must satisfy the stability conditions from the theory of mixed finite element methods; see [11, 12. As is well known, see for example [10, the following two conditions are sufficient:

- $\operatorname{div} \Sigma_{h} \subset V_{h}$.

- There exists a linear operator $\Pi_{h}: H^{1}(\Omega ; \mathbb{S}) \rightarrow \Sigma_{h}$, bounded in $\mathcal{L}\left(H^{1} ; L^{2}\right)$ uniformly with respect to $h$, and such that $\operatorname{div} \Pi_{h} S=\Pi_{h}^{V} \operatorname{div} S$ for all $S \in H^{1}(\Omega ; \mathbb{S})$, where $\Pi_{h}^{V}: L^{2}\left(\Omega ; \mathbb{R}^{3}\right) \rightarrow V_{h}$ denotes the $L^{2}$-projection.

As mentioned above, the construction of finite element spaces that fulfill these two conditions has proved to be surprisingly hard. In this paper, we will derive a family of finite element spaces $\Sigma_{h}$ and $V_{h}$ based on tetrahedral meshes and show that they satisfy these two stability conditions. There is one member of the family for each polynomial degree $k \geq 1$. The space $V_{h}$ for the displacements is simply the space of all piecewise polynomial vector fields of degree at most $k$. In the lowest order case, $k=1$, the space $\Sigma_{h}$ contains the full space of quadratic polynomials 
on each element, augmented by divergence-free polynomials of degrees 3 and 4 . The local dimension of $\Sigma_{h}$ is 162 , or 27 per component of stress on average. The analogous space in two dimensions, derived in [10, was of local dimension 24 (8 per component).

The complexity of the elements may very well limit their practical significance. However, we believe that the determination of the natural discretization of the space $H(\operatorname{div}, \Omega ; \mathbb{S})$ provides important insight, both into the obstacles to the derivation of simpler methods, and for the design of alternative procedures, such as nonconforming methods.

This paper is organized as follows. After giving some preliminaries remarks in Section 2 we present the lowest order element and establish its properties in Section 3. A family of higher order elements is then presented in Section 4 Key to the analysis of these elements is the description of the polynomial space of symmetric matrix fields with vanishing divergence and vanishing normal traces on the boundary of a simplex $K$. The dimension of this space is derived in Section 7 based on preliminary results derived in Sections 5 and 6. Furthermore, an explicit basis for this space, necessary for the computational procedure, is also given in the lowest order cases.

\section{Notation AND PRELIMINARIES}

We begin with some basic notation. If $K \subset \mathbb{R}^{3}$ is a tetrahedron, then $\Delta_{2}(K)$ denotes the set of the four 2-dimensional faces of $K, \Delta_{1}(K)$ the set of the six 1dimensional edges, and $\Delta_{0}(K)$ the set of the four vertices. Furthermore, $\Delta(K)$ is the set of all subsimplices of $K$ (of dimensions $0,1,2$ or 3 ).

We let $\mathbb{M}$ be the space of $3 \times 3$ real matrices, and $\mathbb{S}$ and $\mathbb{K}$ the subspaces of symmetric and skew-symmetric matrices, respectively. The operators sym $: \mathbb{M} \rightarrow \mathbb{S}$ and skw $: \mathbb{M} \rightarrow \mathbb{K}$ denote the symmetric and skew-symmetric parts, respectively. Note that an element of the space $\mathbb{K}$ can be identified with its axial vector in $\mathbb{R}^{3}$ given by the map vec $: \mathbb{K} \rightarrow \mathbb{R}^{3}$ :

$$
\operatorname{vec}\left(\begin{array}{ccc}
0 & -v_{3} & v_{2} \\
v_{3} & 0 & -v_{1} \\
-v_{2} & v_{1} & 0
\end{array}\right)=\left(\begin{array}{l}
v_{1} \\
v_{2} \\
v_{3}
\end{array}\right),
$$

i.e., $\operatorname{vec}^{-1}(v) w=v \times w$ for any vectors $v$ and $w$.

For any vector space $X$, we let $L^{2}(\Omega ; X)$ be the space of square-integrable vector fields on $\Omega$ with values in $X$. For our purposes, $X$ will usually be either $\mathbb{R}, \mathbb{R}^{3}$, or $\mathbb{M}$, or some subspace of one of these. In the case $X=\mathbb{R}$, we will simply write $L^{2}(\Omega)$. The corresponding Sobolev space of order $k$, i.e., the subspace of $L^{2}(\Omega ; X)$ consisting of functions with all partial derivatives of order less than or equal to $k$ in $L^{2}(\Omega ; X)$, is denoted $H^{k}(\Omega ; X)$, and its norm by $\|\cdot\|_{k}$. The space $H(\operatorname{div}, \Omega ; \mathbb{S})$ is defined by

$$
H(\operatorname{div}, \Omega ; \mathbb{S})=\left\{T \in L^{2}(\Omega ; \mathbb{S}) \mid \operatorname{div} T \in L^{2}\left(\Omega ; \mathbb{R}^{3}\right)\right\}
$$

where the divergence of a matrix field is the vector field obtained by applying the divergence operator row-wise. For a vector field $v: \Omega \rightarrow \mathbb{R}^{3}, \operatorname{grad} v$ is the matrix field with rows the gradient of each component, and the symmetric gradient, $\epsilon(v)$, 
is given by $\epsilon(v)=\operatorname{sym} \operatorname{grad} v$. Furthermore,

$$
\operatorname{curl} v=-2 \operatorname{vec} \operatorname{skw} \operatorname{grad} v=\left(\begin{array}{c}
\partial_{3} v_{2}-\partial_{2} v_{3} \\
-\partial_{3} v_{1}+\partial_{1} v_{3} \\
\partial_{2} v_{1}-\partial_{1} v_{2}
\end{array}\right) .
$$

If we consider a linear coordinate transformation of the form $x=B \hat{x}+b$, with corresponding vector fields $v$ and $\hat{v}$ related by $v(x)=\left(B^{\prime}\right)^{-1} \hat{v}(\hat{x})$, then we have

$$
\operatorname{skw}_{\operatorname{grad}} v=\left(B^{\prime}\right)^{-1}\left(\operatorname{skw}_{\operatorname{grad}} \hat{x}\right) B^{-1} .
$$

Here $B^{\prime}$ denotes the transpose of $B$. In particular, if $B$ is orthogonal, i.e., $B^{\prime} B=I$, then $v=B \hat{v}$ and

$$
\text { skw } \operatorname{grad}_{x} v=B\left(\operatorname{skw} \operatorname{grad}_{\hat{x}} \hat{v}\right) B^{\prime} .
$$

As for the divergence and the gradient operator, the operator curl acts on a matrix field by applying the ordinary curl operator to each row of the matrix. The operator curl* is the corresponding operator obtained by taking the curl of each column. Alternatively, we have $\operatorname{curl}^{*} T=\left(\operatorname{curl} T^{\prime}\right)^{\prime}$ for any matrix field $T$. The second order operator curl curl ${ }^{*}$ maps symmetric matrix fields into symmetric matrix fields. Let $\Xi: \mathbb{M} \rightarrow \mathbb{M}$ be the algebraic operator $\Xi T=T^{\prime}-\operatorname{tr}(T) I$, where $I$ is the identity matrix. Then $\Xi$ is invertible with $\Xi^{-1} T=T^{\prime}-\operatorname{tr}(T) I / 2$. The following identities are useful:

$$
\begin{aligned}
\operatorname{vec} \operatorname{skw} \operatorname{curl} T & =-\frac{1}{2} \operatorname{div} \Xi T, \quad T \in C^{\infty}(\Omega, \mathbb{M}), \\
\operatorname{curl} T & =\Xi \operatorname{grad} \operatorname{vec} T, \quad T \in C^{\infty}(\Omega, \mathbb{K}), \\
\operatorname{tr} \operatorname{curl} T & =-2 \operatorname{div} \operatorname{vec} \operatorname{skw} T, \quad T \in C^{\infty}(\Omega, \mathbb{M}) .
\end{aligned}
$$

These formulas can be verified directly, but they are also consequences of the discussions given in [8, 9]; cf. Section 4 of [8] or Section 11 of [9].

For $K \subset \mathbb{R}^{3}$ we let $\mathcal{P}_{k}(K ; X)$ be the space of polynomials of degree $k$, defined on $K$ and with values in $X$. We write $\mathcal{P}_{k}$ or $\mathcal{P}_{k}(K)$ for $\mathcal{P}_{k}(K ; \mathbb{R})$. The de Rham complex has a polynomial analogue of the form

$$
\mathbb{R} \hookrightarrow \mathcal{P}_{k+3} \stackrel{\text { grad }}{\longrightarrow} \mathcal{P}_{k+2}\left(K ; \mathbb{R}^{3}\right) \stackrel{\text { curl }}{\longrightarrow} \mathcal{P}_{k+1}\left(K ; \mathbb{R}^{3}\right) \stackrel{\text { div }}{\longrightarrow} \mathcal{P}_{k} \rightarrow 0 .
$$

In fact, this complex is an exact sequence 9 .

In recent years differential complexes have come to play a significant role in the design of mixed finite element methods [3, 10, 8, 9]. For the equations of elasticity, the relevant differential complex is the elasticity complex. In three space dimensions, the elasticity complex takes the form

$$
\mathbb{T} \hookrightarrow C^{\infty}\left(\Omega ; \mathbb{R}^{3}\right) \stackrel{\epsilon}{\longrightarrow} C^{\infty}(\Omega ; \mathbb{S}) \stackrel{\text { curl curl }^{*}}{\longrightarrow} C^{\infty}(\Omega ; \mathbb{S}) \stackrel{\text { div }}{\longrightarrow} C^{\infty}\left(\Omega ; \mathbb{R}^{3}\right) \rightarrow 0,
$$

where $\mathbb{T}$ is the six-dimensional space of infinitesimal rigid motions, i.e., the space of linear polynomial functions of the form $x \mapsto a+b \times x$ for some $a, b \in \mathbb{R}^{3}$. It is straightforward to verify that the elasticity complex is a complex, i.e., the composition of two successive operators is zero. In fact, if the domain $\Omega$ is contractible, then the elasticity complex is an exact sequence; see [8, 9].

An analogous complex with less smoothness is

$$
\mathbb{T} \hookrightarrow H^{1}\left(\Omega ; \mathbb{R}^{3}\right) \stackrel{\epsilon}{\rightarrow} H\left(\operatorname{curl} \operatorname{curl}^{*}, \Omega ; \mathbb{S}\right) \stackrel{\text { curl curl }}{\longrightarrow} H(\operatorname{div}, \Omega ; \mathbb{S}) \stackrel{\operatorname{div}}{\longrightarrow} L^{2}\left(\Omega ; \mathbb{R}^{3}\right) \rightarrow 0,
$$

where $H\left(\operatorname{curl} \operatorname{curl}^{*}, \Omega ; \mathbb{S}\right)=\left\{S \in L^{2}(\Omega ; \mathbb{S}) \mid \operatorname{curl} \operatorname{curl}^{*} S \in L^{2}(\Omega ; \mathbb{S})\right\}$. 
There is also a polynomial analogue of the elasticity complex. Let $K \subset \mathbb{R}^{3}$ be a tetrahedron and $k \geq 0$. The polynomial elasticity complex is given by

$$
\mathbb{T} \hookrightarrow \mathcal{P}_{k+4}\left(K ; \mathbb{R}^{3}\right) \stackrel{\epsilon}{\rightarrow} \mathcal{P}_{k+3}(K ; \mathbb{S}) \stackrel{\text { curl curl }^{*}}{\longrightarrow} \mathcal{P}_{k+1}(K ; \mathbb{S}) \stackrel{\text { div }}{\longrightarrow} \mathcal{P}_{k}\left(K ; \mathbb{R}^{3}\right) \rightarrow 0 .
$$

This complex is an exact sequence. To prove the exactness, we first show that if $S$ is a matrix field in $\mathcal{P}_{k+3}(\Omega ; \mathbb{S})$, with curl $\operatorname{curl}^{*} S=0$, then $S=\epsilon(u)$ for $u=$ $\left(u_{1}, u_{2}, u_{3}\right)^{T} \in \mathcal{P}_{k+4}\left(\Omega ; \mathbb{R}^{3}\right)$. Clearly $S=\epsilon(u)$ for $u \in C^{\infty}\left(K ; \mathbb{R}^{3}\right)$. It is enough to show that all second derivatives of $u_{k}, k=1,2,3$, are in $\mathcal{P}_{k+2}(\Omega ; \mathbb{R})$. This follows from the identity $\partial_{i j} u_{k}=\partial_{i} \epsilon(u)_{j k}+\partial_{j} \epsilon(u)_{i k}-\partial_{k} \epsilon(u)_{i j}$. Next, we show that if $S \in \mathcal{P}_{k+1}(\Omega ; \mathbb{S})$, and $\operatorname{div} S=0$, then $S=\operatorname{curl} \operatorname{curl}^{*} T$ for some $T \in \mathcal{P}_{k+3}(\Omega ; \mathbb{S})$. First we observe that since $\operatorname{div} S=0$ it follows from the fact that 2.5) is exact that $S=\operatorname{curl} U$ for some $U \in \mathcal{P}_{k+2}(K ; \mathbb{M})$. Furthermore, since $S$ is symmetric it follows from (2.2) that $\operatorname{div} \Xi U=0$, and as a consequence, using (2.5) once more, we obtain that $\Xi U=\operatorname{curl} T$ for some $T \in \mathcal{P}_{k+3}(K ; \mathbb{M})$, or $S=\operatorname{curl} U=\operatorname{curl} \Xi^{-1} \operatorname{curl} T$. However, by (2.3) we have curl $\Xi^{-1}$ curl skw $T=\operatorname{curl} \operatorname{grad} \operatorname{vec} \operatorname{skw} T=0$. Hence, we can take $T \in \mathcal{P}_{k+3}(K ; \mathbb{S})$. Finally, we observe that if $T$ is symmetric, then (2.4) implies that $\operatorname{tr} \operatorname{curl} T=0$, and therefore $S=\operatorname{curl} \Xi^{-1} \operatorname{curl} T=\operatorname{curl}^{\operatorname{curl}{ }^{*}} T$. To establish the surjectivity of the last map, one can use the fact that $\operatorname{dim} \mathcal{P}_{k}=$ $(k+1)(k+2)(k+3) / 6$ to verify that the alternating sum of the dimensions of the spaces in the sequence is zero. The same arguments show that (2.6) is exact for $k=-1,-2$, or -3 , if $\mathcal{P}_{j}$ is interpreted as the zero space for $j<0$.

Let $\left\{\mathcal{T}_{h}\right\}$ denote a family of triangulations of $\Omega$ by tetrahedra with diameter bounded by $h$. We assume that the intersection of any two tetrahedra in $\mathcal{T}_{h}$ is either empty or a common subsimplex of each. The family $\left\{\mathcal{T}_{h}\right\}$ is also assumed to be shape regular in the sense that the ratio of the radii of the circumscribed and inscribed spheres of all the tetrahedra can be bounded by a fixed constant. Furthermore, we will use the notation $\Delta_{j}\left(\mathcal{T}_{h}\right)$, for $j=0,1,2$, to denote the set of vertices, edges, and faces, respectively, associated with the mesh $\mathcal{T}_{h}$. In Section 4 we will define a family of finite element spaces $\Sigma_{h} \subset H(\operatorname{div}, \Omega ; \mathbb{S})$ and $V_{h} \subset L^{2}(\Omega ; \mathbb{R})$ for the elasticity problem consisting of piecewise polynomial spaces with respect to $\mathcal{T}_{h}$ of arbitrarily high polynomial order. However, we will first consider the lowest order case of this family in Section 3 below. All our spaces will have the property that $\operatorname{div} \Sigma_{h} \subset V_{h}$. Furthermore, we will identify a corresponding projection operator $\Pi_{h}: H^{1}(\Omega ; \mathbb{S}) \rightarrow \Sigma_{h}$ satisfying the commutativity relation

$$
\operatorname{div} \Pi_{h} T=\Pi_{h}^{V} \operatorname{div} T, \quad T \in H^{1}(\Omega ; \mathbb{S}),
$$

and the bound

$$
\left\|\Pi_{h} T\right\|_{0} \leq C\|T\|_{1}, \quad T \in H^{1}(\Omega ; \mathbb{S}),
$$

with constant $C$ independent of $h$. Here $\Pi_{h}^{V}: L^{2}\left(\Omega ; \mathbb{R}^{3}\right) \rightarrow V_{h}$ is the $L^{2}$-projection. It is a consequence of the general error bounds derived in [14, cf. also [10, that the properties above imply that $\left(\Sigma_{h}, V_{h}\right)$ is a stable pair of elements for the discretization (1.1), and that the error bounds

$$
\begin{gathered}
\left\|S-S_{h}\right\|_{0} \leq\left\|\left(I-\Pi_{h}\right) S\right\|_{0}, \\
\left\|u-u_{h}\right\|_{0} \leq\left\|\left(I-\Pi_{h}^{V}\right) u\right\|_{0}+c\left\|\left(I-\Pi_{h}\right) S\right\|_{0}
\end{gathered}
$$

hold, with a constant $c$ independent of $h$. Here $(S, u)$ is the unique critical point of the Hellinger-Reissner functional over $H(\operatorname{div}, \Omega ; \mathbb{S}) \times L^{2}\left(\Omega ; \mathbb{R}^{2}\right)$ and $\left(S_{h}, u_{h}\right) \in$ $\Sigma_{h} \times V_{h}$ is the corresponding finite element solution. In addition, $\operatorname{div} S_{h}=\Pi_{h}^{V} \operatorname{div} S$. 


\section{THE LOWEST ORDER ELEMENT}

We first describe the restriction of the lowest order spaces $\Sigma_{h}$ and $V_{h}$ to a single tetrahedron $K \in \mathcal{T}_{h}$. Define

$$
\Sigma_{K}=\left\{T \in \mathcal{P}_{4}(K ; \mathbb{S}) \mid \operatorname{div} T \in \mathcal{P}_{1}\left(K ; \mathbb{R}^{3}\right)\right\}, \quad V_{K}=\mathcal{P}_{1}\left(K ; \mathbb{R}^{3}\right) .
$$

The space $V_{K}$ has dimension 12 and a complete set of degrees of freedom is given by the zero and first order moments with respect to $K$. The space $\Sigma_{K}$ has dimension at least 162 since the dimension of $\mathcal{P}_{4}(K ; \mathbb{S})$ is 210 and the condition $\operatorname{div} T \in \mathcal{P}_{1}\left(K ; \mathbb{R}^{3}\right)$ represents 48 linear constraints. We will show that $\operatorname{dim} \Sigma_{K}=162$ by exhibiting 162 degrees of freedom which determine the elements uniquely. Define

$$
\mathcal{M}(K)=\left\{T \in \mathcal{P}_{4}(K ; \mathbb{S}) \mid \operatorname{div} T=0, T n=0 \quad \text { on } \partial K\right\} .
$$

It will follow from Theorem 7.2 below that the dimension of $\mathcal{M}(K)$ is 6 . Furthermore, in Section 7 we will also give an explicit basis for this space. Using this basis, we can state the 162 degrees of freedom for the space $\Sigma_{K}$.

Lemma 3.1. A matrix field $T \in \Sigma_{K}$ is uniquely determined by the following degrees of freedom:

(1) the values of $T$ at the vertices of $K, 4 \times 6=24$ degrees of freedom,

(2) for each edge $e \in \Delta_{1}(K)$ with unit tangent vector $s$ and linearly independent normal vectors $n_{-}$and $n_{+}$, the constant, linear and quadratic moments over e of $n_{-}^{\prime} T n_{-}, n_{+}^{\prime} T n_{+}, n_{-}^{\prime} T n_{+}, 6 \times 3 \times 3=54$ degrees of freedom,

(3) for each face $f \in \Delta_{2}(K)$, with normal $n$, and each edge e $\subset \partial f$ with tangent $s$, the constant, linear and quadratic moments over e of $s^{\prime} T n, 4 \times 3 \times 3=36$ degrees of freedom,

(4) for each face $f \in \Delta_{2}(K)$, with normal $n$, the constant and linear moments over $f$ of $T n, 4 \times 3 \times 3=36$ degrees of freedom,

(5) the average of $T$ over $K, 6$ degrees of freedom,

(6) the value of the moments $\int_{K} T: U d x, U \in \mathcal{M}(K), 6$ degrees of freedom.

Proof. We assume that all degrees of freedom vanish and show that $T=0$. Since $T=0$ at the vertices, the second and third set of degrees of freedom imply that $T n=0$ on each edge for both faces meeting the edge. By the fourth set of degrees of freedom we obtain that $T n=0$ on each face of $K$. For $v=\operatorname{div} T \in \mathcal{P}_{1}\left(K ; \mathbb{R}^{3}\right)$ we have

$$
\int_{K} v^{2} d x=-\int_{K} T: \epsilon(v) d x+\int_{\partial K} T n \cdot v d x_{f}=-\int_{K} T: \epsilon(v) d x=0
$$

by the fifth set of degrees of freedom. Here and below, $d x_{f}$ denotes the surface measure on $\partial K$. We conclude that $\operatorname{div} T=0$, and, by the last set of degrees of freedom, that $T=0$.

We now describe the finite element spaces on the triangulation $\mathcal{T}_{h}$. We denote by $V_{h}$ the space of vector fields that belong to $V_{K}$ for each $K \in \mathcal{T}_{h}$ and by $\Sigma_{h}$ the space of matrix fields that belong piecewise to $\Sigma_{K}$, and with the continuity conditions induced by the degrees of freedom. In particular, for $T \in \Sigma_{h}$, the normal components $T n$ are continuous across all faces $f \in \Delta_{2}\left(\mathcal{T}_{h}\right)$ and, hence, $\Sigma_{h} \subset H(\operatorname{div}, \Omega ; \mathbb{S})$. In addition, if $T \in \Sigma_{h}, e \in \Delta_{1}\left(\mathcal{T}_{h}\right)$, and $n_{+}, n_{-}$are two vectors normal to $e$, then $n_{+}^{\prime} T n_{-}$is continuous on $e$ and, of course, $T$ is continuous at the vertices. 
It remains to define an interpolation operator $\Pi_{h}: H^{1}(\Omega ; \mathbb{S}) \rightarrow \Sigma_{h}$ that satisfies (2.7) and (2.8). The technique used here is standard and can be found for example in [12, $\S$ VI-4]. Because of the vertex and edge degrees of freedom, the canonical interpolation operator for $\Sigma_{h}, \Pi_{h}^{\Sigma}$, defined directly from the degrees of freedom, is not bounded on $H^{1}(\Omega ; \mathbb{S})$. In order to overcome this difficulty we introduce the operator $\Pi_{h}^{0}: H^{1}(\Omega ; \mathbb{S}) \rightarrow \Sigma_{h}$ defined from the degrees of freedom above, but where the vertex and edge degrees of freedom are set equal to zero, i.e., we have

$$
\begin{gathered}
\Pi_{h}^{0} T(x)=0, \quad x \in \Delta_{0}\left(\mathcal{T}_{h}\right), \\
\int_{e} n_{-}^{\prime} \Pi_{h}^{0} T n_{+} v d s=0, \quad e \in \Delta_{1}\left(\mathcal{T}_{h}\right), v \in \mathcal{P}_{2}(e), \text { where } n_{-}, n_{+} \in e^{\perp}, \\
\int_{e} s^{\prime} \Pi_{h}^{0} T n v d s=0, \quad v \in f \in \Delta_{2}\left(\mathcal{T}_{h}\right), e \in \Delta_{1}(f), n \perp f, s \| e, v \in \mathcal{P}_{2}(e), \\
\int_{f}\left(T-\Pi_{h}^{0} T\right) n \cdot v d x_{f}=0, \quad f \in \Delta_{2}\left(\mathcal{T}_{h}\right), v \in \mathcal{P}_{1}\left(f ; \mathbb{R}^{3}\right), \\
\int_{K}\left(T-\Pi_{h}^{0} T\right) d x=0, \quad K \in T_{h}, \\
\int_{K}\left(T-\Pi_{h}^{0} T\right): U d x=0, \quad K \in T_{h}, U \in \mathcal{M}(K) .
\end{gathered}
$$

The commutativity property (2.7) for $\Pi_{h}^{0}$ follows from (3.4) and (3.5) since

$$
\int_{K}\left(\operatorname{div} \Pi_{h}^{0} T-\operatorname{div} T\right) \cdot v d x=-\int_{K}\left(\Pi_{h}^{0} T-T\right): \epsilon(v) d x+\int_{\partial K}\left(\Pi_{h}^{0} T-T\right) n \cdot v d x_{f}=0 .
$$

The uniform boundedness (2.8) can be seen from a standard scaling argument using the matrix Piola transform. Let $\hat{K}$ be a fixed reference tetrahedron and $F=F_{K}: \hat{K} \rightarrow K$ be an affine isomorphism of the form $F \hat{x}=B \hat{x}+b$. Given a matrix field $\hat{T}: \hat{K} \rightarrow \mathbb{S}$, define $T: K \rightarrow \mathbb{S}$ by the matrix Piola transform $T(x)=B \hat{T}(\hat{x}) B^{T}$, with $x=F \hat{x}$. Using $\operatorname{div} T(x)=B \operatorname{div} \hat{T}(\hat{x})$, it is easy to verify that $T \in \Sigma_{K}$ if and only if $\hat{T} \in \Sigma_{\hat{K}}$. Furthermore, as in [4, 10], a scaling argument can be used to verify the uniform boundedness condition (2.8) for the operator $\Pi_{h}^{0}$. We can therefore conclude that the operator $\Pi_{h}^{0}$ satisfies the two conditions (2.7) and (2.8). However, the operator $\Pi_{h}^{0}$ lacks good approximation properties. Therefore, in order to obtain error estimates from the general bounds (2.9) and (2.10) a more accurate interpolation operator is needed.

Consider the modified interpolation operator $\Pi_{h}: H^{1}(\Omega ; \mathbb{S}) \rightarrow \Sigma_{h}$ of the form

$$
\Pi_{h}=\Pi_{h}^{0}\left(I-R_{h}\right)+R_{h},
$$

where $R_{h}: L^{2}(\Omega ; \mathbb{S}) \rightarrow \Sigma_{h}$ is the Clément operator onto the continuous piecewise quadratic subspace of $\Sigma_{h}$ [13]. This operator satifies the bounds

$$
\left\|R_{h} T-T\right\|_{j} \leq c h^{m-j}\|T\|_{m}, \quad 0 \leq j \leq 1, \quad j \leq m \leq 3 .
$$

As a consequence of this bound, and the boundedness (2.8) of $\Pi_{h}^{0}$, we obtain the estimate

$$
\left\|\Pi_{h} T-T\right\|_{0} \leq c h^{m}\|T\|_{m}, \quad 1 \leq m \leq 3
$$

for the interpolation error. Furthermore, since $\Pi_{h}^{0}$ satisfies (2.7) and $\Pi_{h}^{V} \operatorname{div} R_{h}=$ $\operatorname{div} R_{h}$, we conclude that $\Pi_{h}$ satisfies (2.7). 
We also recall that the projection operator $\Pi_{h}^{V}: L^{2}\left(\Omega ; \mathbb{R}^{3}\right) \rightarrow V_{h}$ satisfies the error estimate

$$
\left\|\Pi_{h}^{V} v-v\right\|_{0} \leq c h^{m}\|v\|_{m}, \quad 0 \leq m \leq 2 .
$$

The estimates (3.8) and (3.9), combined with the basic error bounds (2.9) and (2.10), and the fact that $\operatorname{div} S_{h}=\Pi_{h}^{V} \operatorname{div} S$, imply the following error estimates for the finite element method generated by $\Sigma_{h} \times V_{h}$.

Theorem 3.2. Let $(S, u)$ denote the unique critical point of the Hellinger-Reissner functional over $H(\operatorname{div}, \Omega ; \mathbb{S}) \times L^{2}\left(\Omega ; \mathbb{R}^{2}\right)$ and let $\left(S_{h}, u_{h}\right)$ be the unique critical point over $\Sigma_{h} \times V_{h}$. Then

$$
\begin{array}{rlrl}
\left\|S-S_{h}\right\|_{0} & \leq c h^{m}\|S\|_{m}, & 1 \leq m \leq 3, \\
\left\|\operatorname{div} S-\operatorname{div} S_{h}\right\|_{0} & \leq c h^{m}\|\operatorname{div} S\|_{m}, \quad 0 \leq m \leq 2, \\
\left\|u-u_{h}\right\|_{0} & \leq c h^{m}\|u\|_{m+1}, & 1 \leq m \leq 2 .
\end{array}
$$

Remark. It is not possible to lower the polynomial degree of the stress space $\Sigma_{h}$ introduced above. That is, if $\Sigma_{K}=\left\{T \in \mathcal{P}_{k}(K ; \mathbb{S}) \mid \operatorname{div} T \in \mathcal{P}_{s}\left(K ; \mathbb{R}^{3}\right)\right\}$, then we must require that $k \geq 4$ and $s \leq k-3$. The argument generalizes a similar argument given in [10]. Any element $T \in \Sigma_{K}$ must be uniquely determined by an arbitrary specification of the degrees of freedom that are associated with the vertices, edges, faces, and interior of $K$. Since we require the assembled finite element space to be contained in $H(\operatorname{div}, \Omega ; \mathbb{S})$, the degrees of freedom associated with a face, its edges, and its vertices must determine $T n$ on that face. Now, consider two faces of $K$ meeting an edge $e$ with normals $n_{+}$and $n_{-}$. The quantity $n_{-}^{\prime} T n_{+}$is determined on $e$ by the degrees of freedom associated to the first face and its edges and vertices, and, since this quantity is identical to $n_{+}^{\prime} T n_{-}$, it is also determined by the degrees of freedom associated to the second face and its edges and vertices. But, by definition, the degrees of freedom are independent, so this is only possible if $n_{-}^{\prime} T n_{+}$is determined on $e$ by the degrees of freedom associated to $e$ or associated to one of its two vertices. But the edge $e$ is shared by other simplices which will have different values for the normals $n_{+}$and $n_{-}$, from which it follows that the degrees of freedom associated to $e$ and those associated to its two vertices must determine the quantities $m^{\prime} T n$ for every pair of vectors $m, n \in e^{\perp}$ (assuming there is no restriction put on the triangulations). Next, consider a vertex $x$ belonging to an edge $e$ and a face with normal $n$. Then we have just seen that $n^{\prime} T(x) n$ is determined by the degrees of freedom associated to $e$ and its vertices. But it is similarly determined by the corresponding quantities for the other edge in the face containing $x$. By independence of the degrees of freedom, we conclude that $n^{\prime} T(x) n$ is determined by the degrees of freedom associated with $x$. This is true for each face containing $x$, so for arbitrary values of $n$. It follows that $T$ itself is determined at a vertex by the degrees of freedom associated to the vertex (a symmetric matrix is determined by the associated quadratic form).

Moreover, for the commutativity relation (2.7) to hold, we need to have as degrees of freedom on a face the moment of $T n$ times the divergence of the stress elements. Since we already have enough degrees of freedom at the vertices and on edges to determine two components of $T n$ there, we can give at most the moments of degree $k-3$ of these components. Thus our polynomial space must incorporate the restriction $s \leq k-3$. It follows that $k<3$ is impossible. For $k=3$, the above argument leads to 24 degrees of freedom at the vertices and 36 degrees of 
freedom on the edges. Next, for each face, and each edge, we take as degrees of freedom the constant and linear moments of $s^{\prime} T n, 24$ degrees of freedom, as well as the constant moments of $T n$ on faces, which are another 12 degrees of freedom for $H$ (div) continuity. In total we have 96 degrees of freedom, but the space of cubics with constant divergence has dimension 93, from the exactness of (2.6). It follows that $k \geq 4$.

However, as in [10, a minor simplification is possible. On each tetrahedron $K \in \mathcal{T}_{h}$ we take the restricted displacement space $V_{K}$ to be the rigid motions $\mathbb{T} \subset \mathcal{P}_{1}\left(K ; \mathbb{R}^{3}\right)$ and the corresponding stress space to be

$$
\tilde{\Sigma}_{K}=\left\{T \in \mathcal{P}_{4}(K ; \mathbb{S}) \mid \operatorname{div} T \in \mathbb{T}\right\} .
$$

Clearly $\operatorname{dim} \tilde{\Sigma}_{K} \geq 210-(60-6)=156$. In fact, $\operatorname{dim} \tilde{\Sigma}_{K}=156$, and a complete set of degrees of freedom is obtained by removing the six average values of $T$ represented by (4) in Lemma 3.1. The proof of the fact that these degrees of freedom are unisolvent for $\tilde{\Sigma}_{K}$ follows by a simple modification of the proof of Lemma 3.1 above. Just observe that if $v=\operatorname{div} T \in \mathbb{T}$, then $\epsilon(v)=0$. However, the simplified element is less accurate, since the stress space lacks some quadratics, and the displacement space lacks some linears. Instead of the error estimates given in Theorem 3.2 we obtain at most $O\left(h^{2}\right)$ convergence for $\left\|S-S_{h}\right\|_{0}$, and at most first order convergence for $\left\|\operatorname{div}\left(S-S_{h}\right)\right\|_{0}$ and $\left\|u-u_{h}\right\|_{0}$.

\section{A FAMiLy of Higher ORDER ELEMENTS}

In this section we describe a family of stable element pairs, one for each degree $k \geq 1$. The lowest order case $k=1$ is the one treated above. We first describe the elements on a single tetrahedron. Define

$$
\Sigma_{K}=\left\{T \in \mathcal{P}_{k+3}(K ; \mathbb{S}) \mid \operatorname{div} T \in \mathcal{P}_{k}\left(K ; \mathbb{R}^{3}\right)\right\}, \quad V_{K}=\mathcal{P}_{k}\left(K ; \mathbb{R}^{3}\right) .
$$

Then

$$
\begin{aligned}
& \operatorname{dim} V_{K}=3\left(\begin{array}{c}
k+3 \\
3
\end{array}\right)=\frac{(k+3)(k+2)(k+1)}{2} \\
\operatorname{dim} \Sigma_{K} \geq d_{k}:= & \operatorname{dim} \mathcal{P}_{k+3}(K ; \mathbb{S})-\left[\operatorname{dim} \mathcal{P}_{k+2}\left(K ; \mathbb{R}^{3}\right)-\operatorname{dim} \mathcal{P}_{k}\left(K ; \mathbb{R}^{3}\right)\right] \\
= & 6\left(\begin{array}{c}
k+6 \\
3
\end{array}\right)-3\left(\begin{array}{c}
k+5 \\
3
\end{array}\right)+3\left(\begin{array}{c}
k+3 \\
3
\end{array}\right)=k^{3}+12 k^{2}+56 k+93 .
\end{aligned}
$$

Notice that the space $\epsilon\left[\mathcal{P}_{k}\left(K ; \mathbb{R}^{3}\right)\right]$ has dimension $(k+3)(k+2)(k+1) / 2-6$. Analoguous to the lowest order case, we define the space

$$
\mathcal{M}_{k}(K)=\left\{T \in \mathcal{P}_{k}(K ; \mathbb{S}) \mid \operatorname{div} T=0, \quad T n=0 \quad \text { on } \partial K\right\} .
$$

We will prove in Section 7 Theorem 7.2, that $\operatorname{dim} \mathcal{M}_{k}(K)$ is $(k+2)(k-2)(k-3) / 2$ for $k \geq 4$.

The degrees of freedom for $V_{K}$ are the moments of degree less than or equal to $k$ with respect to $K$. A unisolvent set of degrees of freedom for $\Sigma_{K}$ is given by

(1) the values of $T$ at the vertices of $K, 4 \times 6=24$ degrees of freedom,

(2) for each edge $e \in \Delta_{1}(K)$ with unit tangent vector $s$ and linearly independent normal vectors $n_{-}$and $n_{+}$, the moments of degree at most $k+1$ over $e$ of $n_{-}^{\prime} T n_{-}, n_{+}^{\prime} T n_{+}, n_{-}^{\prime} T n_{+}, 6 \times(k+2) \times 3=18 k+36$ degrees of freedom, 
(3) for each face $f \in \Delta_{2}(K)$, with normal $n$, and each edge $e \subset \partial f$ with tangent $s$, the moments of degree at most $k+1$ over $e$ of $s^{\prime} T n, 4 \times 3 \times(k+2)=12 k+24$ degrees of freedom,

(4) for each $f \in \Delta_{2}(K)$ with normal vector $n$, the moments of degree at most $k$ over $f$ for $T n, 3 \times 4 \times(k+2)(k+1) / 2=6 k^{2}+18 k+12$ degrees of freedom,

(5) $\int_{K} T: U d x, U \in \epsilon\left(V_{K}\right),(k+3)(k+2)(k+1) / 2-6$ degrees of freedom,

(6) $\int_{K} T: U d x, U \in \mathcal{M}_{k+3}(K),(k+5)(k+1) k / 2$ degrees of freedom.

The proof that this set of functionals is unisolvent for the space $\Sigma_{K}$ is almost identical to the lowest order case, and it is easily checked that their numbers sum up to $d_{k}$. Hence, we have shown that $\operatorname{dim} \Sigma_{k}=d_{k}$. Furthermore, in Section 7 we will give an explicit basis for the space $\mathcal{M}_{k}(K)$ when $k=4$ and $k=5$.

The finite element space $V_{h} \subset L^{2}\left(\Omega ; \mathbb{R}^{3}\right)$ consists of all vector fields that belong to $\mathcal{P}_{k}\left(K ; \mathbb{R}^{3}\right)$ for each $K \in \mathcal{T}_{h}$, while the corresponding stress space $\Sigma_{h}$ is the space of matrix fields that belong piecewise to $\Sigma_{K}$, and with the continuity conditions induced by the degrees of freedom. In particular, this implies that the normal components $T n$, for $T \in \Sigma_{h}$, are continuous over all faces in $\Delta_{2}\left(\mathcal{T}_{h}\right)$. Hence, as in the lowest order case we have that $\Sigma_{h} \subset H(\operatorname{div}, \Omega ; \mathbb{S})$.

The $L^{2}$-projection $\Pi_{h}^{V}$ onto $V_{h}$ satisfies the estimate

$$
\left\|\Pi_{h}^{V} v-v\right\|_{0} \leq c h^{m}\|v\|_{m}, \quad 0 \leq m \leq k+1 .
$$

We also introduce the Clément interpolant $R_{h}: L^{2}(\Omega ; \mathbb{S}) \rightarrow \Sigma_{h}$ defined as the $\mathbb{S}$ valued version of the standard scalar Clément interpolant into continuous piecewise polynomials of order $k+1$. Hence, the operator $R_{h}$ satisfies

$$
\left\|R_{h} T-T\right\|_{j} \leq c h^{m-j}\|T\|_{m}, \quad 0 \leq j \leq 1, j \leq m \leq k+2 .
$$

Furthermore, we define the modified canonical interpolation operator $\Pi_{h}$ by (3.7), where the operator $\Pi_{h}^{0}$ is defined in complete analogy with the lowest order case, by setting the degrees of freedom associated with $\Delta_{0}\left(\mathcal{T}_{h}\right)$ and $\Delta_{1}\left(\mathcal{T}_{h}\right)$ equal to zero. Then the operator $\Pi_{h}$ satisfies (2.7) and (2.8), and the error bound

$$
\left\|\Pi_{h} T-T\right\|_{0} \leq c h^{m}\|T\|_{m}, \quad 1 \leq m \leq k+2 .
$$

As above, the interpolation estimates (4.1) and (4.2), and the error bounds (2.9) and (2.10), lead to the following error estimates.

Theorem 4.1. Let $(S, u)$ denote the unique critical point of the Hellinger-Reissner funtional over $H(\operatorname{div}, \Omega ; \mathbb{S}) \times L^{2}\left(\Omega ; \mathbb{R}^{2}\right)$ and let $\left(S_{h}, u_{h}\right)$ be the unique critical point over $\Sigma_{h} \times V_{h}$. Then

$$
\begin{gathered}
\left\|S-S_{h}\right\|_{0} \leq c h^{m}\|S\|_{m}, \quad 1 \leq m \leq k+2, \\
\left\|\operatorname{div} S-\operatorname{div} S_{h}\right\|_{0} \leq c h^{m}\|\operatorname{div} S\|_{m}, \quad 0 \leq m \leq k+1, \\
\left\|u-u_{h}\right\|_{0} \leq c h^{m}\|u\|_{m+1}, \quad 1 \leq m \leq k+1
\end{gathered}
$$

\section{Some PROperties OF VECTOR FIELDS AND MATRIX FIELDS}

It remains to prove the claimed dimension formula for $\mathcal{M}_{k}(K)$, given in Theo rem 7.2 below. To do so we introduce a related space, $\mathcal{N}_{k}(K)$, in the next section and determine its dimension. For the analysis we need some basic notations and properties of differential operators on vector fields and matrix fields, which are the subject of the present section. 
5.1. Identities for vector fields. Let $n$ denote a fixed unit vector in $\mathbb{R}^{3}, P_{n}=$ $n n^{\prime}$ the orthogonal projection onto $\mathbb{R} n, f=n^{\perp}$ the plane orthogonal to $n$, and $Q_{n}=I-P_{n}$ the orthogonal projection onto $f$. Furthermore, set

$$
C_{n}=\left(\begin{array}{ccc}
0 & n_{3} & -n_{2} \\
-n_{3} & 0 & n_{1} \\
n_{2} & -n_{1} & 0
\end{array}\right)=-\operatorname{vec}^{-1} n,
$$

so that $C_{n} v=v \times n$. The following identities are easily checked:

$$
C_{n}^{\prime}=-C_{n}, \quad C_{n}^{2}=-Q_{n}, \quad C_{n} P_{n}=0, \quad C_{n} Q_{n}=Q_{n} C_{n}=C_{n} .
$$

For any vector field $v=\left(v_{1}, v_{2}, v_{3}\right)^{\prime}$ in $\mathbf{R}^{3}$, we obviously have $v=P_{n} v+Q_{n} v$ and $\operatorname{curl} v=\operatorname{curl} P_{n} v+\operatorname{curl} Q_{n} v=P_{n} \operatorname{curl} P_{n} v+P_{n} \operatorname{curl} Q_{n} v+Q_{n} \operatorname{curl} P_{n} v+Q_{n} \operatorname{curl} Q_{n} v$. It is elementary to verify that

$$
P_{n} \operatorname{curl} P_{n} v=0, \quad Q_{n} \operatorname{curl} Q_{n} v=C_{n} \frac{\partial v}{\partial n}
$$

if $n=e_{3}=(0,0,1)^{\prime}$. In view of the transformation formula (2.1), these identities hold for an arbitrary unit vector $n$. Furthermore, we define

$$
\operatorname{rot}_{f} v=P_{n} \operatorname{curl} v=P_{n} \operatorname{curl} Q_{n} v=-\left(\operatorname{div} C_{n} v\right) n, \quad \operatorname{curl}_{f} v=Q_{n} \operatorname{curl} P_{n} v .
$$

With this notation we obtain the decomposition

$$
\operatorname{curl} v=\operatorname{rot}_{f} v+\operatorname{curl}_{f} v+C_{n} \frac{\partial v}{\partial n} .
$$

We also define the tangential gradient $\operatorname{grad}_{f} \phi=Q_{n} \operatorname{grad} \phi$, for a scalar field $\phi$. For a vector field $v, \operatorname{grad}_{f} v=(\operatorname{grad} v) Q_{n}$ is the matrix field with rows equal to the tangential gradients of the components of $v$, and we let

$$
\epsilon_{f}(v)=\frac{1}{2}\left\{\operatorname{grad}_{f}\left(Q_{n} v\right)+\left[\operatorname{grad}_{f}\left(Q_{n} v\right)\right]^{\prime}\right\}=Q_{n} \epsilon(v) Q_{n}
$$

be the tangential part of the symmetric gradient. Note that the definitions of $\operatorname{grad}_{f} v, \epsilon_{f}(v), \operatorname{curl}_{f} v$, and $\operatorname{rot}_{f} v$ do not depend on the choice of the unit normal $n$ to $f$. The identity

$$
\operatorname{curl}_{f} v=-C_{n} \operatorname{grad}\left(n^{\prime} v\right)=-C_{n} \operatorname{grad}_{f}\left(n^{\prime} v\right)
$$

can be easily verified in the special case $n=e_{3}$ and holds in general.

5.2. Identities for matrix fields. We extend the operators curl, $\operatorname{curl}_{f}$ and $\operatorname{rot}_{f}$ to act on and yield $3 \times 3$ matrix fields by applying the vector operations row-wise. More precisely, $\operatorname{rot}_{f} S=(\operatorname{curl} S) P_{n}=\left(\operatorname{curl} S Q_{n}\right) P_{n}$ and $\operatorname{curl}_{f} S=\left(\operatorname{curl} S P_{n}\right) Q_{n}$. We notice that, for any constant matrix $A, \operatorname{curl} A S=A \operatorname{curl} S$. We also recall that curl $^{*} S=\left(\operatorname{curl} S^{\prime}\right)^{\prime}$ is the corresponding operator obtained by applying the curl operation to each column. The corresponding column operators rot ${ }_{f}^{*}$ and curl $_{f}^{*}$ are defined similarly, i.e., $\operatorname{rot}_{f}^{*} S=P_{n} \operatorname{curl}^{*} S=P_{n} \operatorname{curl}^{*} Q_{n} S$ and $\operatorname{curl}_{f}^{*} S=$ $Q_{n} \operatorname{curl}^{*} P_{n} S$. For a given row vector $v, \operatorname{grad}_{f}^{*} v=\left(\operatorname{grad}_{f} v^{\prime}\right)^{\prime}=Q_{n}\left(\operatorname{grad} v^{\prime}\right)^{\prime}$, which is the matrix whose columns are the tangential gradients of the components of $v$.

We now extend the decomposition (5.1) to curl and $\operatorname{curl}^{*}$. It is easy to see that $C_{n} S$ results in $C_{n}$ applied to the columns of $S$, while $-S C_{n}$ is $C_{n}$ applied row-wise. It follows that

$$
\operatorname{curl} S=\operatorname{curl}_{f} S+\operatorname{rot}_{f} S-\frac{\partial S}{\partial n} C_{n}
$$


and

$$
\operatorname{curl}^{*} S=\operatorname{curl}_{f}^{*} S+\operatorname{rot}_{f}^{*} S+C_{n} \frac{\partial S}{\partial n}
$$

where $\partial S / \partial n$ is obtained by taking the directional derivative of each component. Furthermore, the identities

$$
\operatorname{curl}_{f} S=\left(\operatorname{grad}_{f} S n\right) C_{n}, \quad \operatorname{curl}_{f}^{*} S=-C_{n} \operatorname{grad}_{f}^{*} n^{\prime} S
$$

are just matrix analogues of (5.2). Note also that from the definitions of the operators $\operatorname{rot}_{f}$ and $\operatorname{rot}_{f}^{*}$ we have

$$
\begin{aligned}
P_{n}\left(\operatorname{curl}_{\operatorname{curl}^{*}} S\right) P_{n} & =P_{n}\left(\operatorname{rot}_{f} \operatorname{curl}^{*} S\right)=\operatorname{rot}_{f}\left(P_{n} \operatorname{curl}^{*} S\right) \\
& =\operatorname{rot}_{f} \operatorname{rot}_{f}^{*} S=\operatorname{rot}_{f} \operatorname{rot}_{f}^{*}\left(Q_{n} S Q_{n}\right) .
\end{aligned}
$$

We will also need exact sequences relating spaces of functions defined on a twodimensional space. Let $f=n^{\perp}$. In analogy with (2.6) the following two-dimensional complexes are exact:

$$
\mathbb{T}_{f} \hookrightarrow \mathcal{P}_{k+3}\left(f ; Q_{n} \mathbb{R}^{3}\right) \stackrel{\epsilon_{f}}{\longrightarrow} \mathcal{P}_{k+2}\left(f ; Q_{n} \mathbb{S} Q_{n}\right) \stackrel{\operatorname{rot}_{f} \operatorname{rot}_{f}^{*}}{\longrightarrow} \mathcal{P}_{k}\left(f ; \mathbb{R} P_{n}\right) \rightarrow 0,
$$

$$
\mathcal{P}_{1}(f ; \mathbb{R}) \hookrightarrow \mathcal{P}_{k+3}(f ; \mathbb{R}) \stackrel{\operatorname{grad}_{f} \operatorname{grad}_{f}^{*}}{\longrightarrow} \mathcal{P}_{k+1}\left(f ; Q_{n} \mathbb{S} Q_{n}\right) \stackrel{\operatorname{rot}_{f}}{\longrightarrow} \mathcal{P}_{k}\left(f ; Q_{n} \mathbb{S} P_{n}\right) \rightarrow 0 .
$$

Here, $\mathbb{T}_{f}$ is the 3 -dimensional space of vector fields on $f$ of the form $v(x)=Q_{n} w(x)$ for some $w \in \mathbb{T}$.

To close this section, we introduce a useful operator $\Lambda_{f}$. Let $f$ be a plane with unit normal $n$. For any symmetric matrix field $S$ defined on a neighborhood on $f$, we define $\Lambda_{f}(S): f \rightarrow Q_{n} \mathbb{S} Q_{n}$ by

$$
\Lambda_{f}(S)=2 \epsilon_{f}(S n)-Q_{n} \partial_{n} S Q_{n},
$$

where $\partial_{n} S:=\partial S / \partial n$. Hence, $\Lambda_{f}(S)$ is a tangential symmetric matrix field defined on $f$. Note that $\Lambda_{f}(S)$ depends on the choice of normal: if we reverse the sign of $n$, we reverse the sign of $\Lambda_{f}(S)$ as well. For future reference, we note that if $T=\epsilon(v)$, where $v$ is a vector field then we have

$$
2 \epsilon_{f}(T n)=\operatorname{grad}_{f} \operatorname{grad}_{f}^{*}\left(n^{\prime} v\right)+Q_{n} \partial_{n} \epsilon(v) Q_{n} .
$$

Hence, we obtain that

$$
\Lambda_{f}(\epsilon(v))=\operatorname{grad}_{f} \operatorname{grad}_{f}^{*}\left(n^{\prime} v\right) .
$$

The tangential-normal components of the matrix field curlcurl ${ }^{*} S$ on $f$ can be expressed in terms of $\Lambda_{f}(S)$. Indeed, by the definition of the operator $\operatorname{rot}_{f}$ and (5.3) we have

$$
C_{n}\left(\operatorname{curl} \operatorname{curl}^{*} S\right) P_{n}=C_{n} \operatorname{rot}_{f} \operatorname{curl}^{*} S=\operatorname{rot}_{f} C_{n}\left(\operatorname{curl}_{f}^{*} S+\operatorname{rot}_{f}^{*} S+C_{n} \partial_{n} S\right) .
$$

However, $C_{n} \operatorname{rot}_{f}^{*} S=0$ and, by (5.3),$C_{n} \operatorname{curl}_{f}^{*} S=Q_{n} \operatorname{grad}_{f}^{*} n^{\prime} S$. Hence,

$$
C_{n}\left(\operatorname{curlcurl}^{*} S\right) P_{n}=\operatorname{rot}_{f} Q_{n}\left(\operatorname{grad}_{f}^{*} n^{\prime} S-\partial_{n} S\right) Q_{n}=\operatorname{rot}_{f} \Lambda_{f}(S) .
$$




\section{Polynomial matrix Fields on a Single tetrahedron}

In this section we will compute the dimension of the polynomial space

$$
\mathcal{N}_{k}=\mathcal{N}_{k}(K)=\left\{S \in \mathcal{P}_{k}(K ; \mathbb{S}) \mid Q_{n} S Q_{n}=\Lambda_{f}(S)=0, f \in \Delta_{2}(K)\right\},
$$

where $K \subset \mathbb{R}^{3}$ is a fixed tetrahedron and $k \geq 3$. The final result is obtained in Theorem [6.6, and applied in the next section to obtain the dimension of the space $\mathcal{M}_{k}(K)$. We start by introducing some additional notation.

If $f \in \Delta_{2}(K)$, we denote by $h_{f}$ the perpendicular distance from the opposite vertex to $f$ and by $n=n_{f}$ the outward normal vector to $f$. If $e$ is an edge, we let $s=s_{e}$ denote one of the unit vectors parallel to $e$. When the edge $e$ belongs to the face $f$, we write $m=m_{e, f}$ for the unit vector in $f$, normal to $e$, pointing from $e$ into $f$. See Figure 1 . When the notation $f_{+}$and $f_{-}$is used to denote two faces, the corresponding normals will be denoted $n_{+}$and $n_{-}$, and the perpendicular distances $h_{+}$and $h_{-}$, respectively. The notation $m_{+}$and $m_{-}$will also be used to denote $m_{e, f_{+}}$and $m_{e, f_{-}}$where $e$ is the edge common to $f_{+}$and $f_{-}$.

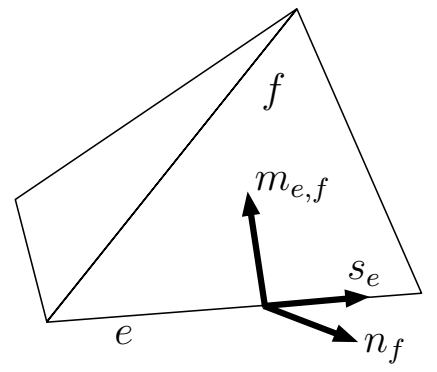

Figure 1 . The $\left(n_{f}, s_{e}, m_{e, f}\right)$ coordinate system for a face $f$ and edge $e$ of the tetrahedron $K$.

The barycentric coordinates on $K$ will be labelled by the faces. That is, they are $\lambda_{f} \in \mathcal{P}_{1}(K ; \mathbb{R})$ determined by $\lambda_{f} \equiv 0$ on $f$ and $\sum_{f} \lambda_{f} \equiv 1$ on $K$. We recall that $\operatorname{grad} \lambda_{f}=-n_{f} / h_{f}$. Let $g \in \Delta(K)$ be a face of dimension $m$ with vertices $x_{i_{0}}, x_{i_{1}}, \ldots, x_{i_{m}}$. For $m=0, g$ is a vertex, for $m=1, g$ is an edge, and so on. We define the bubble functions $b_{g}=\lambda_{f_{i_{0}}} \lambda_{f_{i_{1}}} \cdots \lambda_{f_{i_{m}}}$, where $f_{i_{k}}$ is the face opposite the vertex $x_{i_{k}}$. For $d>0, \mathcal{P}_{d}^{g}(K)=\operatorname{span}\left\{\lambda_{f_{i_{0}}}^{j_{0}} \lambda_{f_{i_{1}}}^{j_{1}} \cdots \lambda_{f_{i_{m}}}^{j_{m}} \mid j_{0}+\cdots+j_{m}=d\right\}$, so that $\operatorname{dim} \mathcal{P}_{d}^{g}(K)=\operatorname{dim} \mathcal{P}_{d}(g)$. Note that if $x$ is a vertex opposite face $f$, then $b_{x}=\lambda_{f}$ and $\mathcal{P}_{d}^{x}(K)=\mathbb{R} \lambda_{f}^{d}$, while $b_{K}=\prod_{f} \lambda_{f}$ and $\mathcal{P}_{d}^{K}=\mathcal{P}_{d}$. For a given face $f_{0} \in \Delta_{2}(K)$, $b_{f_{0}}=b_{K} / \lambda_{f_{0}}=\prod_{f \neq f_{0}} \lambda_{f}$, and for a given edge $e \in \Delta_{1}(K), b_{e}=b_{K} /\left(\lambda_{f_{-}} \lambda_{f_{+}}\right)$, where $f_{-}$and $f_{+}$are the faces containing $e$.

The monomials of degree $k$ in the barycentric coordinates form a basis for $\mathcal{P}_{k}(K)$, and by grouping together terms according to which coordinates enter the monomial, we can uniquely represent any $p \in \mathcal{P}_{k}$ as

$$
p=\sum_{g \in \Delta(K)} b_{g} p_{g}, \quad p_{g} \in \mathcal{P}_{k-1-\operatorname{dim} g}^{g}(K) .
$$

The standard Lagrangian degrees of freedom for $p \in \mathcal{P}_{k}(K)$ are the values of $p$ at the vertices, the moments of $p$ of degree at most $k-2$ on each of the edges of $K$, the moments of degree at most $k-3$ on each of the faces, and the moments of degree 
at most $k-4$ on $K$. From the vertex values of $p$ we may determine the polynomials $p_{g}$ in (6.2) for $g \in \Delta_{0}(K)$. From these and the edge moments we may determine as well the $p_{g}$ for $g \in \Delta_{1}(K)$, etc.

Of course analogous considerations apply to $\mathcal{P}_{k}(K ; X)$ for $X$ a vector space. In particular, we have the representation $p=\sum_{g \in \Delta(K)} b_{g} p_{g}$ for $p \in \mathcal{P}_{k}(K ; X)$ where now $p_{g} \in \mathcal{P}_{k-1-\operatorname{dim} g}^{g}(K ; X)$, the space of $X$-valued polynomials on $K$ whose components with respect to a basis of $X$ belong to $\mathcal{P}_{k-1-\operatorname{dim} g}^{g}(K)$.

If $e=f_{-} \cap f_{+} \in \Delta_{1}(K)$, with $f_{-}, f_{+} \in \Delta_{2}(K)$, we let $G_{e} \in \mathbb{S}$ be the matrix

$$
G_{e}=n_{-} n_{+}^{\prime}+n_{+} n_{-}^{\prime} \text {. }
$$

We note that $G_{e} s=0$ and $m_{-}^{\prime} G_{e} m_{-}=m_{+}^{\prime} G_{e} m_{+}=0$.

Lemma 6.1. For $k \geq 0$, the dimension of the space

$$
\mathcal{N}_{k}^{0}=\mathcal{N}_{k}^{0}(K):=\left\{S \in \mathcal{P}_{k}(K ; \mathbb{S}) \mid Q_{n} S Q_{n}=0, \quad f \in \Delta_{2}(K)\right\}
$$

is $(k+1) k(k-1)$.

Proof. For a subsimplex $g \in \Delta(K)$, let us first define

$$
N^{g}=\left\{S \in \mathbb{S} \mid Q_{n} S Q_{n}=0 \text { for all faces containing } g\right\} .
$$

Clearly, if $g=K$, then $\operatorname{dim} N^{g}=6$, if $g \in \Delta_{2}(K)$, then $\operatorname{dim} N^{g}=3$, and if $g \in \Delta_{0}(K)$, then $\operatorname{dim} N^{g}=0$. Finally, if $g \in \Delta_{1}(K)$, i.e., $g=e$ is an edge, then $\operatorname{dim} N^{g}=1$. In fact, the space $N^{e}$ is then spanned by the matrix $G_{e}$ introduced above.

If $S \in \mathcal{N}_{k}^{0}$, then from (6.2) we obtain the representation

$$
S=\sum_{g \in \Delta(K)} b_{g} S_{g}, \quad S_{g} \in \mathcal{P}_{k-1-\operatorname{dim} g}^{g}\left(K ; N^{g}\right) .
$$

As a consequence

$$
\begin{aligned}
\operatorname{dim} \mathcal{N}_{k}^{0} & =\sum_{g \in \Delta(K)} \operatorname{dim} \mathcal{P}_{k-1-\operatorname{dim} g}^{g}\left(K ; N^{g}\right) \\
& =6(k-1)+6(k-1)(k-2)+(k-1)(k-2)(k-3)=(k+1) k(k-1) .
\end{aligned}
$$

Before we are able to compute $\operatorname{dim} \mathcal{N}_{k}$, we need to establish several lemmas.

Lemma 6.2. If $S \in \mathcal{N}_{k}$, then $S$ is zero on each edge.

Proof. As above let $e=f_{-} \cap f_{+} \in \Delta_{1}(K)$, with $f_{-}, f_{+} \in \Delta_{2}(K)$. Then

$$
m_{-}^{\prime} n_{+}=m_{+}^{\prime} n_{-}<0 \text {. }
$$

In fact, the transformation

$$
\left(\begin{array}{c}
m_{-} \\
n_{-}
\end{array}\right) \mapsto\left(\begin{array}{c}
n_{+} \\
m_{+}
\end{array}\right)
$$

is a rotation in the plane orthogonal to $e$.

Let $S \in \mathcal{N}_{k}$. Since $\mathcal{N}_{k} \subset \mathcal{N}_{k}^{0}$ we know that $\left.S\right|_{e}=\rho G_{e}$, where $\rho \in \mathcal{P}_{k}(e)$. Therefore, if we can show that

$$
m_{+}^{\prime} S n_{+}+m_{-}^{\prime} S n_{-}=0
$$

on $e$, then $\rho\left(m_{+}^{\prime} n_{-}+m_{-}^{\prime} n_{+}\right)=2 \rho m_{+}^{\prime} n_{-}=0$, and, as a consequence, $S$ is zero on $e$. It therefore suffices to show (6.5). 
On $e$, we must have $s^{\prime} \Lambda_{f}(S) m=0$, i.e.,

$$
\partial_{s}\left(m^{\prime} S n\right)+\partial_{m}\left(s^{\prime} S n\right)-\partial_{n}\left(s^{\prime} S m\right)=0
$$

where $f$ is either $f_{-}$or $f_{+}$. By adding this property for the two faces we obtain

$$
\begin{aligned}
-\partial_{s}\left(m_{+}^{\prime} S n_{+}\right. & \left.+m_{-}^{\prime} S n_{-}\right) \\
& =\left[\partial_{m_{-}}\left(s^{\prime} S n_{-}\right)-\partial_{n_{-}}\left(s^{\prime} S m_{-}\right)\right]-\left[\partial_{n_{+}}\left(s^{\prime} S m_{+}\right)-\partial_{m_{+}}\left(s^{\prime} S n_{+}\right)\right] .
\end{aligned}
$$

However, the right-hand side here is zero as a consequence of the fact that the transformation (6.4) is a rotation. In fact, this property implies that

$$
\partial_{m_{-}}\left(v^{\prime} n_{-}\right)-\partial_{n_{-}}\left(v^{\prime} m_{-}\right)=\partial_{n_{+}}\left(v^{\prime} m_{+}\right)-\partial_{m_{+}}\left(v^{\prime} n_{+}\right)
$$

for any smooth vector field $v$ on $K$. Hence, we can conclude that $m_{+}^{\prime} S n_{+}+m_{-}^{\prime} S n_{-}$ is a constant along $e$, and since it is zero at the vertices, (6.5) holds.

Lemma 6.3. Let

$$
\begin{aligned}
\mathcal{N}_{k, \partial K}:=\left\{U \in \mathcal{N}_{k}^{0} \mid U=\right. & \sum_{\substack{f \in \Delta_{2}(K)\\
}} b_{f} U_{f}, U_{f} \in \mathcal{P}_{k-3}^{f}(K ; \mathbb{S}) \text { and } \\
& \left.\left.\Lambda_{f}(U)\right|_{e}=0 \text { for each face } f \text { and each edge } e \text { of } f\right\} .
\end{aligned}
$$

Then $\operatorname{dim} \mathcal{N}_{k, \partial K}=6\left(k^{2}-6 k+10\right)$.

Proof. If $U=\sum_{f} b_{f} U_{f}$, then $U \in \mathcal{N}_{k}^{0}$ if and only if each coefficient $U_{f} \in \mathcal{P}_{k-3}^{f}(K ; \mathbb{S})$ satisfies

$$
Q_{n} U_{f} Q_{n}=0 \quad \text { on } f \text {. }
$$

Hence, this property is assumed to hold. We have $\Lambda_{f}(U)=0$ on an edge $e \subset f$ if and only if the three terms $s^{\prime} \Lambda_{f}(U) s, s^{\prime} \Lambda_{f}(U) m$ and $m^{\prime} \Lambda_{f}(U) m$ vanish there. For any fixed unit vector $t$ and $e \in \Delta_{1}(K)$, we have

$$
\partial_{t} U=\sum_{f}\left(\partial_{t} b_{f}\right) U_{f}=-b_{e}\left(\frac{t^{\prime} n_{+}}{h_{+}} U_{f_{-}}+\frac{t^{\prime} n_{-}}{h_{-}} U_{f_{+}}\right) \quad \text { on } e,
$$

where $f_{-}$and $f_{+}$are the two faces meeting the edge $e$ and we have used that $\operatorname{grad} b_{f_{-}}=-n_{+} b_{e} / h_{+}$on $e$.

Recall that $s^{\prime} \Lambda_{f}(U) s=2 \partial_{s}\left(s^{\prime} U n\right)-\partial_{n}\left(s^{\prime} U s\right)$. Since $U=0$ on $e$,

$$
s^{\prime} \Lambda_{f}(U) s=-\partial_{n}\left(s^{\prime} U s\right) \quad \text { on } e .
$$

However, since $Q_{n} U_{f} Q_{n}=0$ on the face $f$, we have $s^{\prime} U_{f_{-}} s=s^{\prime} U_{f_{+}} s=0$ on $e$. By (6.6), with $t=n$, we conclude that $s^{\prime} \Lambda_{f}(U) s=0$ on $e$.

Next, similar considerations for $s^{\prime} \Lambda_{f}(U) m=\partial_{s}\left(m^{\prime} U n\right)+\partial_{m}\left(s^{\prime} U n\right)-\partial_{n}\left(s^{\prime} U m\right)$ give

$$
s^{\prime} \Lambda_{f_{+}}(U) m_{+}=\partial_{m_{+}}\left(s^{\prime} U n_{+}\right)-\partial_{n_{+}}\left(s^{\prime} U m_{+}\right) \quad \text { on } e .
$$

Furthermore, from (6.6) we have

$$
\partial_{m_{+}}\left(s^{\prime} U n_{+}\right)=-m_{+}^{\prime} n_{-} b_{e} \frac{s^{\prime} U_{f_{+}} n_{+}}{h_{-}}
$$

on $e$, and, using $s^{\prime} U_{f_{-}} m_{-}=s^{\prime} U_{f_{+}} m_{+}=0$, we obtain

$$
\partial_{n_{+}}\left(s^{\prime} U m_{+}\right)=-b_{e} \frac{s^{\prime} U_{f_{-}} m_{+}}{h_{+}}=-m_{+}^{\prime} n_{-} b_{e} \frac{s^{\prime} U_{f_{-}} n_{-}}{h_{+}} .
$$


It follows that

$$
s^{\prime} \Lambda_{f_{+}}(U) m_{+}=m_{+}^{\prime} n_{-} b_{e}\left(\frac{s^{\prime} U_{f_{-}} n_{-}}{h_{+}}-\frac{s^{\prime} U_{f_{+}} n_{+}}{h_{-}}\right) \text {on } e .
$$

We have therefore shown that $s^{\prime} \Lambda_{f}(U) m=0$ on all edges if and only if

$$
\frac{s^{\prime} U_{f_{-}} n_{-}}{h_{+}}=\frac{s^{\prime} U_{f_{+}} n_{+}}{h_{-}}
$$

on all edges of $K$.

Finally, we consider $m^{\prime} \Lambda_{f}(U) m=2 \partial_{m}\left(m^{\prime} U n\right)-\partial_{n}\left(m^{\prime} U m\right)$. Using the fact that both $m_{-}^{\prime} U_{f_{-}} m_{-}$and $m_{+}^{\prime} U_{f_{+}} m_{+}$vanish on $e$, we obtain from (6.6) that, on $e$,

$$
\begin{aligned}
\partial_{n_{+}} m_{+}^{\prime} U m_{+} & =-b_{e} \frac{m_{+}^{\prime} U_{f_{-}} m_{+}}{h_{+}} \\
& =-\frac{b_{e}}{h_{+}}\left[\left(m_{+}^{\prime} n_{-}\right)^{2} n_{-}^{\prime} U_{f_{-}} n_{-}+2 m_{+}^{\prime} m_{-} m_{+}^{\prime} n_{-} m_{-}^{\prime} U_{f_{-}} n_{-}\right],
\end{aligned}
$$

SO

$$
m_{+}^{\prime} \Lambda_{f_{+}}(U) m_{+}=m_{+}^{\prime} n_{-} b_{e}\left[m_{+}^{\prime} n_{-} \frac{n_{-}^{\prime} U_{f_{-}} n_{-}}{h_{+}}-2\left(\frac{m_{+}^{\prime} U_{f_{+}} n_{+}}{h_{-}}-m_{+}^{\prime} m_{-} \frac{m_{-}^{\prime} U_{f_{-}} n_{-}}{h_{+}}\right)\right] .
$$

Hence, $m_{+}^{\prime} \Lambda_{f_{+}}(U) m_{+}$vanishes on $e$ if and only if

$$
n_{-}^{\prime} U_{f_{-}} n_{-}=\frac{2 h_{+}}{m_{+}^{\prime} n_{-}}\left(\frac{m_{+}^{\prime} U_{f_{+}} n_{+}}{h_{-}}-m_{+}^{\prime} m_{-} \frac{m_{-}^{\prime} U_{f_{-}} n_{-}}{h_{+}}\right) \text {on } e .
$$

Note that this condition is not symmetric in $f_{-}$and $f_{+}$. We thus obtain two conditions for each edge $e$.

Since $U_{f} \in \mathcal{P}_{k-3}^{f}(K ; \mathbb{S})$, with $Q_{n} U_{f} Q_{n}=0$ on $f$, it follows that $U_{f}$ is uniquely determined by the vector field $v_{f}:=U_{f} n \in \mathcal{P}_{k-3}\left(f ; \mathbb{R}^{3}\right)$. The analysis above shows that $U=\sum_{f} b_{f} U_{f} \in \mathcal{N}_{k, \partial K}$ if and only if these vector fields satisfy

$$
\begin{aligned}
& \text { (A) } \frac{s^{\prime} v_{f_{-}}}{h_{+}}=\frac{s^{\prime} v_{f_{+}}}{h_{-}} \text {on } e, \text { and } \\
& \text { (B) } n_{-}^{\prime} v_{f_{-}}=\frac{2 h_{+}}{m_{+}^{\prime} n_{-}}\left(\frac{m_{+}^{\prime} v_{f_{+}}}{h_{-}}-m_{+}^{\prime} m_{-} \frac{m_{-}^{\prime} v_{f_{-}}}{h_{+}}\right) \text {on } e,
\end{aligned}
$$

whenever an edge $e$ is shared by faces $f_{-}$and $f_{+}$. Therefore, there is an isomorphism between $\mathcal{N}_{k, \partial K}$ and

$$
\left\{\left(v_{f}\right) \in \prod_{f \in \Delta_{2}(K)} \mathcal{P}_{k-3}\left(f ; \mathbb{R}^{3}\right) \mid \text { the } v_{f} \text { satisfy }(\mathrm{A}) \text { and }(\mathrm{B})\right\} .
$$

To compute the dimension of the space (6.8) we consider the relations (A) and (B) at a fixed vertex $x$ of $K$. If $\left(v_{f}\right)$ is an element of the space (6.8), define $z \in \mathbb{R}^{3}$ by

$$
s^{\prime} z=h_{f} s^{\prime} v_{f}(x)
$$

for $s$ chosen as tangents to each edge $e$ meeting $x$, and where $f$ is a face meeting $e$. Note that the vector $z$ is well defined as a consequence of condition (A), and that for each face $f$ containing $x$ we have $h_{f} t^{\prime} v_{f}(x)=t^{\prime} z$ for all vectors $t$ that are tangential to the face $f$. Using the expansion $n_{-}=\left[m_{+}-\left(m_{+}^{\prime} m_{-}\right) m_{-}\right] / m_{+}^{\prime} n_{-}$we can then rewrite condition (B) at the vertex $x$ as

$$
h_{f} n_{f}^{\prime} v_{f}(x)=2 n_{f}^{\prime} z \text {. }
$$


From this discussion we can conclude that the dimension of the space (6.8), and hence $\operatorname{dim} \mathcal{N}_{k, \partial K}$, is at least $6\left(k^{2}-6 k+10\right)$. To see this observe that

$$
\operatorname{dim} \prod_{f} \mathcal{P}_{k-3}\left(f, \mathbb{R}^{3}\right)=6(k-2)(k-1) .
$$

Furthermore, the conditions (A) and (B) represent a total of $6 \cdot 3 \cdot(k-4)=18(k-4)$ constraints in the interior of the edges and $4 \cdot 6=24$ constraints at the vertices. Since $6(k-2)(k-1)-18(k-4)-24=6\left(k^{2}-6 k+10\right)$, this is a lower bound for $\operatorname{dim} \mathcal{N}_{k, \partial K}$.

We complete the proof by showing that elements of the space (6.8) are determined by $6\left(k^{2}-6 k+10\right)$ degrees of freedom, in fact by degrees of freedom corresponding to the space

$$
\prod_{x \in \Delta_{0}(K)} \mathbb{R}^{3} \times \prod_{e \in \Delta_{1}(K)} \mathcal{P}_{k-5}\left(e ; \mathbb{R}^{3}\right) \times \prod_{f \in \Delta_{2}(K)} \mathcal{P}_{k-6}\left(f ; \mathbb{R}^{3}\right) .
$$

To see this, for each vertex $x$ pick a vector $z=z(x) \in \mathbb{R}^{3}$ and choose $v_{f}(x)$ such that the relations (6.9) and (6.10) hold for all faces meeting $x$. This determines the vectors $v_{f}(x)$ for all vertices $x \in \partial f$. We then define $h_{f} s^{\prime} v_{f}$ on each edge by the standard interior degrees of freedom, and $m^{\prime} v_{f}$ with respect to both faces meeting $e$ are determined similarly. These degrees of freedom on the edges correspond to the space $\prod_{e} \mathcal{P}_{k-5}\left(e ; \mathbb{R}^{3}\right)$. The normal components $n_{f}^{\prime} v_{f}$ are determined on each edge by condition (B). Finally, we must apply the interior degrees of freedom to $v_{f}$ on $f$. We conclude that elements of the space $\mathcal{N}_{k, \partial K}$ are determined by $12+18 \operatorname{dim} \mathcal{P}_{k-5}(e)+12 \operatorname{dim} \mathcal{P}_{k-6}(f)=6\left(k^{2}-6 k+10\right)$ degrees of freedom.

For $U \in \mathcal{N}_{k, \partial K}$ and $f \in \Delta_{2}(K), \Lambda_{f}(U)$ is a polynomial vanishing on $\partial f$, and so the quotient $\Lambda_{f}(U) / b_{f}$ is a polynomial. We define $T_{f}: \mathcal{N}_{k, \partial K} \rightarrow \mathcal{P}_{k-4}\left(f, Q_{n} \mathbb{S} Q_{n}\right)$ by

$$
T_{f}(U)=-h_{f} \Lambda_{f}(U) / b_{f} .
$$

Lemma 6.4. If $f_{-}, f_{+} \in \Delta_{2}(K), e=f_{-} \cap f_{+}$, and $s$ is a unit vector parallel to $e$, then

$$
s^{\prime} T_{f_{+}}(U) s=s^{\prime} T_{f_{-}}(U) s \quad \text { on } e, \quad U \in \mathcal{N}_{k, \partial K} .
$$

Proof. First we show that

$$
\partial_{m_{+}} s^{\prime} \Lambda_{f_{+}}(U) s=\partial_{m_{-}} s^{\prime} \Lambda_{f_{-}}(U) s \quad \text { on } e .
$$

Recall that $\partial_{m_{+}} b_{f_{+}}=-m_{+}^{\prime} n_{-} b_{e} / h_{-}, \partial_{m_{-}} b_{f_{-}}=-m_{-}^{\prime} n_{+} b_{e} / h_{+}$on $e$ and $m_{+}^{\prime} n_{-}=$ $m_{-}^{\prime} n_{+}$. We have on an edge $e, \partial_{m} s^{\prime} \Lambda_{f}(U) s=2 \partial_{s} \partial_{m} s^{\prime} U n-\partial_{m} \partial_{n} s^{\prime} U s$. Using (6.6) we obtain

$$
\partial_{m_{+}} s^{\prime} U n_{+}=-b_{e} \frac{m_{+}^{\prime} n_{-}}{h_{-}} s^{\prime} U_{f_{+}} n_{+},
$$

which is symmetric in $f_{-}$and $f_{+}$as a consequence of (6.7) and $m_{+}^{\prime} n_{-}=m_{-}^{\prime} n_{+}$.

The identity (6.11) will follow if we show that $\partial_{m_{+}} \partial_{n_{+}} s^{\prime} U s=\partial_{m_{-}} \partial_{n_{-}} s^{\prime} U s$. Consider first the term

$$
V=b_{f_{-}} U_{f_{-}}+b_{f_{+}} U_{f_{+}} .
$$


Since $Q_{n} U_{f} Q_{n}=0$ for $f=f_{-}, f_{+}$and $\operatorname{grad} b_{f_{-}}=-\frac{b_{e}}{h_{+}} n_{+}$on $f_{+}$we derive that at the edge $e$,

$$
\begin{aligned}
\partial_{m_{+}} \partial_{n_{+}} s^{\prime} V s & =-b_{e}\left[\frac{1}{h_{+}} s^{\prime} \partial_{m_{+}} U_{f_{-}} s+\frac{m_{+}^{\prime} n_{-}}{h_{-}} s^{\prime} \partial_{n_{+}} U_{f_{+}} s\right] \\
& =-\left(m_{+}^{\prime} n_{-}\right) b_{e}\left[\frac{1}{h_{+}} s^{\prime} \partial_{n_{-}} U_{f_{-}} s+\frac{1}{h_{-}} s^{\prime} \partial_{n_{+}} U_{f_{+}} s\right],
\end{aligned}
$$

and this expression is symmetric in $f_{-}$and $f_{+}$. Finally, consider terms of the form $W=b_{f} U_{f}$, where $f$ is neither $f_{-}$nor $f_{+}$. In this case $b_{f}=\lambda_{f_{-}} \lambda_{f_{+}} \lambda$, where $\lambda$ is the barycentic coordinate associated with the fourth face $\left(\neq f, f_{-}, f_{+}\right)$of $K$, and on $e$ we have

$$
\partial_{m_{+}} \partial_{n_{+}} s^{\prime} W s=\frac{m_{+}^{\prime} n_{-}}{h_{-} h_{+}} \lambda s^{\prime} U_{f} s .
$$

This is again symmetric in $f_{-}$and $f_{+}$. We have therefore established (6.11).

Now, by definition, $h_{+} \Lambda_{f_{+}}(U)=-b_{f_{+}} T_{f_{+}}(U)$. Therefore

$$
\left(m_{+}^{\prime} n_{-}\right)^{-1} h_{+} h_{-} \partial_{m_{+}} s^{\prime} \Lambda_{f_{+}}(U) s=b_{e} s^{\prime} T_{f_{+}}(U) s \quad \text { on } e .
$$

By (6.11), the left-hand side is unchanged if we interchange the subscripts + and - , so the same must be true of the right-hand side.

Lemma 6.5. Let $\left(T_{f}\right) \in \prod_{f \in \Delta_{2}(K)} \mathcal{P}_{k}\left(f ; Q_{n} \mathbb{S} Q_{n}\right)$ be such that $s^{\prime} T_{f_{-}} s=s^{\prime} T_{f_{+}} s$ on $e$, whenever $e=f_{-} \cap f_{+}, f_{-}, f_{+} \in \Delta_{2}(K)$. Then there exists an $S \in \mathcal{P}_{k}(K ; \mathbb{S})$ such that $Q_{n} S Q_{n}=T_{f}$ for all $f \in \Delta_{2}(K)$.

Proof. We will define $S \in \mathcal{P}_{k}(K ; \mathbb{S})$ by first specifying its vertex values, then specifying its moments of degree at most $k-2$ on the edges, then its moments of degree at most $k-3$ on the faces, and then the moments of degree at most $k-4$ over the interior of $K$.

Let $x$ be a vertex. We define the matrix $S(x) \in \mathbb{S}$ by specifying the values $s_{i}^{\prime} S(x) s_{j}$ where the $s_{i}$ are the tangents to the edges $e_{i}$ meeting at $x$ (and so the $s_{i}$ form a basis for $\mathbb{R}^{3}$ ). Namely we take $s_{i}^{\prime} S(x) s_{j}=s_{i}^{\prime} T_{f} s_{j}$ with $f$ the face containing $e_{i}$ and $e_{j}$. If $i=j$ there are two possible choices of the face $f$, but they give the same result by assumption.

For the interior degrees of freedom on an edge $e$ we use the basis $s, m_{-}, m_{+}$of $\mathbb{R}^{3}$, and let $T_{e} \in \mathcal{P}_{k}(e ; \mathbb{S})$ be given by

$$
\begin{gathered}
s^{\prime} T_{e} s=s^{\prime} T_{f_{-}} s=s^{\prime} T_{f_{+}} s, \quad s^{\prime} T_{e} m_{-}=s^{\prime} T_{f_{-}} m_{-}, \quad s^{\prime} T_{e} m_{+}=s^{\prime} T_{f_{+}} m_{+}, \\
m_{-}^{\prime} T_{e} m_{-}=m_{-}^{\prime} T_{f_{-}} m_{-}, \quad m_{+}^{\prime} T_{e} m_{+}=m_{+}^{\prime} T_{f_{+}} m_{+}, \quad m_{-}^{\prime} T_{e} m_{+}=0 .
\end{gathered}
$$

Then we define $\left.S\right|_{e}$ by

$$
\int_{e}\left(S-T_{e}\right) V d s=0, \quad V \in \mathcal{P}_{k-2}(e ; \mathbb{S}) .
$$

Similarly, for the interior degrees of freedom on each face we let $Q_{n} S Q_{n}$ inherit the moments from $T_{f}$, while the data for $S P_{n}$ is taken to be zero. The interior degrees of freedom on $K$ are all taken to be zero.

As a consequence of the two previous lemmas, there is a map

$$
\mathcal{N}_{k, \partial K} \rightarrow \mathcal{P}_{k-4}(K ; \mathbb{S}), \quad U \mapsto S(U),
$$

such that $Q_{n} S(U) Q_{n}=T_{f}(U)$ for all faces $f \in \Delta_{2}(K)$.

We are finally ready to compute the dimension of the space $\mathcal{N}_{k}$ defined in (6.1). 
Theorem 6.6. For $k \geq 3$, the dimension of the space $\mathcal{N}_{k}$ is $k\left(k^{2}-6 k+11\right)$.

Proof. Let $S \in \mathcal{N}_{k}$. By Lemma 6.2, $S$ must be zero on each edge and so can be written $S=\sum_{f} b_{f} S_{f}+b_{K} S_{K}$, where $S_{f} \in \mathcal{P}_{k-3}^{f}(K ; \mathbb{S})$ and $S_{K} \in \mathcal{P}_{k-4}(K ; \mathbb{S})$. Now $\epsilon_{f}\left(b_{K} S_{K} n_{f}\right)$ vanishes on $f$ since $b_{K}$ does, while

$$
\partial_{n}\left(b_{K} Q_{n} S_{K} Q_{n}\right)=\left(\partial_{n} b_{K}\right) Q_{n} S_{K} Q_{n}=-\frac{b_{f}}{h_{f}} Q_{n} S_{K} Q_{n} \quad \text { on } f .
$$

Thus

$$
\Lambda_{f}\left(b_{K} S_{K}\right)=\frac{b_{f}}{h_{f}} Q_{n} S_{K} Q_{n} .
$$

In particular, $\Lambda_{f}\left(b_{K} S_{K}\right)$ vanishes on $\partial f$. It follows that if $S \in \mathcal{N}_{k}$ and we define $U=\sum_{f} b_{f} S_{f}$, then $U \in \mathcal{N}_{k, \partial K}$. Therefore, the map $\left(U, S_{K}\right) \mapsto U+b_{K} S_{K}$ defines an isomorphism from

$$
\left\{\left(U, S_{K}\right) \in \mathcal{N}_{k, \partial K} \times \mathcal{P}_{k-4}(K ; \mathbb{S}) \mid Q_{n} S_{K} Q_{n}=T_{f}(U) \text { on each face } f\right\}
$$

onto $\mathcal{N}_{k}$.

Finally, note that a matrix field of the form $b_{K} V, V \in \mathcal{P}_{k-4}(K ; \mathbb{S})$, belongs to $\mathcal{N}_{k}$ if and only if $V \in \mathcal{N}_{k-4}^{0}$. Therefore, using the map (6.12), the mapping

$$
\mathcal{N}_{k, \partial K} \times \mathcal{N}_{k-4}^{0} \rightarrow \mathcal{N}_{k}, \quad(U, V) \mapsto(U, S(U)+V),
$$

is an isomorphism. It follows that $\operatorname{dim} \mathcal{N}_{k}=\operatorname{dim} \mathcal{N}_{k, \partial K}+\operatorname{dim} \mathcal{N}_{k-4}^{0}$ and using Lemma 6.3 and Lemma 6.1 we get $\operatorname{dim} \mathcal{N}_{k}=6\left(k^{2}-6 k+10\right)+(k-3)(k-4)(k-5)=$ $k\left(k^{2}-6 k+11\right)$.

\section{THE SPACE OF DIVERGENCE-FREE MATRIX FIELDS WITH VANISHING NORMAL TRACES}

Recall that the space

$$
\mathcal{M}_{k}=\mathcal{M}_{k}(K)=\left\{S \in \mathcal{P}_{k}(K ; \mathbb{S}) \mid \operatorname{div} S=0 \quad \text { on } K, \quad P_{n} S=0, \quad f \in \Delta_{2}(K)\right\}
$$

appears in the degrees of freedom for the finite element space $\Sigma_{h} \subset H(\operatorname{div}, \Omega ; \mathbb{S})$ introduced in Sections 3 and 4 Therefore, a derivation of the dimension of this space is fundamental for our theory, while a construction of a (dual) basis for the space $\mathcal{M}_{k}$ is necessary for the implementation of the method. The dimension formula will be a simple consequence of the following lemma, in which $\mathcal{P}_{k+3}^{0}\left(K ; \mathbb{R}^{3}\right):=\{v \in$ $\mathcal{P}_{k+3}\left(K ; \mathbb{R}^{3}\right) \mid v \equiv 0$ on $\left.\partial K\right\}=b_{K} \mathcal{P}_{k-1}\left(K ; \mathbb{R}^{3}\right)$.

Lemma 7.1. (1) The operator curl curl ${ }^{*}$ maps $\mathcal{N}_{k+2}(K)$ onto $\mathcal{M}_{k}(K)$.

(2) $\left\{T \in \mathcal{N}_{k+2} \mid \operatorname{curl} \operatorname{curl}^{*} T=0\right\}=\epsilon\left[\mathcal{P}_{k+3}^{0}\left(K ; \mathbb{R}^{3}\right)\right]$.

(3) The following sequence is exact:

$$
0 \rightarrow \mathcal{P}_{k+3}^{0}\left(K ; \mathbb{R}^{3}\right) \stackrel{\epsilon}{\rightarrow} \mathcal{N}_{k+2}(K) \stackrel{\text { curl curl }}{\longrightarrow} \mathcal{M}_{k}(K) \rightarrow 0 .
$$

Proof. It follows directly from (5.5) and (5.9) that curl $\operatorname{curl}^{*} \mathcal{N}_{k+2} \subset \mathcal{M}_{k}$. Hence, to prove the first statement we need only show that $\mathcal{M}_{k} \subset \operatorname{curl}^{\operatorname{curl}^{*}} \mathcal{N}_{k+2}$. Let $S \in \mathcal{M}_{k}$. Since $\operatorname{div} S=0$, it follows from the exactness of the complex (2.6) that there is a $T \in \mathcal{P}_{k+2}(K ; \mathbb{S})$ such that $S=\operatorname{curl} \operatorname{curl}^{*} T$. The proof will be completed by constructing a vector field $u \in \mathcal{P}_{k+3}\left(K ; \mathbb{R}^{3}\right)$ such that

$$
Q_{n}(T-\epsilon(u)) Q_{n}=0, \quad \Lambda_{f}(T-\epsilon(u))=0, \quad \text { on each face } f .
$$


Note that since $S \in \mathcal{M}_{k}$ it follows from (5.5) that

$$
\operatorname{rot}_{f} \operatorname{rot}_{f}^{*} Q_{n} T Q_{n}=P_{n}\left(\operatorname{curlcurl}{ }^{*} T\right) P_{n}=P_{n} S P_{n}=0 \text { on each face } f .
$$

Hence, from the exact sequence (5.6) we conclude that for each face $f \in \Delta_{2}(K)$ there is a vector field $v_{f} \in \mathcal{P}_{k+3}\left(f ; \mathbb{R}^{3}\right)$, with $P_{n} v_{f}=0$, such that $Q_{n} T Q_{n}=$ $\epsilon_{f}\left(v_{f}\right)$. The vector fields $v_{f}$ are uniquely determined up to a $2 \mathrm{D}$ rigid motion, and hence we may normalize them so that $\int_{e} s^{\prime} v_{f} d s=0$ on each edge $e \subset f$. Since $\partial_{s}\left(s^{\prime} v_{v_{f_{-}}}\right)=s^{\prime} T s=\partial_{s}\left(s^{\prime} v_{f_{+}}\right)$on each edge, we obtain that $P_{s} v_{f_{-}}=P_{s} v_{f_{+}}$ on each edge $e=f_{+} \cap f_{-}$. As a consequence, there is a $v \in \mathcal{P}_{k+3}\left(K ; \mathbb{R}^{3}\right)$ such that $Q_{n} v Q_{n}=v_{f}$ on each face $f$. Then $Q_{n} \epsilon(v) Q_{n}=\epsilon_{f}\left(v_{f}\right)=Q_{n} T Q_{n}$, i.e., $Q_{n} U Q_{n}=0$ on each face $f$, where $U=T-\epsilon(v)$. This implies, in particular, that $U s$ and $\operatorname{grad}\left(s^{\prime} U s\right)$ vanish on each edge $e \in \Delta_{1}(K)$. Therefore,

$$
s^{\prime} \Lambda_{f}(U) s=2 \partial_{s}\left(n^{\prime} U s\right)-\partial_{n}\left(s^{\prime} U s\right)=0 \quad \text { on } \partial f
$$

for each face $f$.

Next, observe that, by (5.9), $\operatorname{rot}_{f} \Lambda_{f}(U)=C_{n} S P_{n}=0$. Hence, (5.7) implies that there is a scalar field $q_{f} \in \mathcal{P}_{k+4}(f ; \mathbb{R})$, uniquely determined up to a linear function on $f$, such that $\Lambda_{f}(U)=\operatorname{grad}_{f} \operatorname{grad}_{f}^{*} q_{f}$. On each edge $e \in \partial f$, we have by (7.3) that $0=s^{\prime} \Lambda_{f}(U) s=\partial_{s}^{2} q_{f}$. It follows that we can assume that $q_{f} \equiv 0$ on $\partial f$. Hence, there exists another vector field $w$ in $\mathcal{P}_{k+3}\left(K ; \mathbb{R}^{3}\right)$ such that $Q_{n} w=0$ and $n^{\prime} w=q_{f}$ on each face. Recall by (5.8) that $\Lambda_{f}(\epsilon(w))=\operatorname{grad}_{f} \operatorname{grad}_{f}^{*} q_{f}=\Lambda_{f}(U)$. Hence, if we let $u=v+w$, then the relation (7.2) holds. This proves the first statement.

We now prove the second statement. If $T=\epsilon(v)$ for some $v \in \mathcal{P}_{k+3}^{0}\left(K ; \mathbb{R}^{3}\right)$, then $\operatorname{curl} \operatorname{curl}^{*} T=0$, and, by (5.8),

$$
Q_{n} T Q_{n}=\epsilon_{f}\left(Q_{n} v\right), \quad \Lambda_{f}(T)=\operatorname{grad}_{f} \operatorname{grad}_{f}^{*}\left(n^{\prime} v\right) \quad \text { on } f,
$$

for each face $f$. Since $v$ vanishes on $f$, the right-hand sides of these equations vanish, and so $T$ belongs to $\mathcal{N}_{k+2}(K)$. Conversely, if $T \in \mathcal{N}_{k+2}(K)$, then, by the exactness of the sequence (2.6), $T=\epsilon(v)$ for some $v \in \mathcal{P}_{k+3}\left(K ; \mathbb{R}^{3}\right)$, which is determined uniquely if we require that

$$
\int_{e} s^{\prime} v d s=0, \quad e \in \Delta_{1}(K)
$$

(The functionals $v \mapsto \int_{e} s^{\prime} v d s, e \in \Delta_{1}(K)$, form a set of degrees of freedom for $\mathbb{T}$, the null space of $\epsilon$.) From the first equation in (7.4) and (7.5), we find that $Q_{n} v$ vanishes on each face $f$. Therefore the entire vector $v$ vanishes on each edge $e$. Using the second equation in (7.4), we see that $n^{\prime} v$ vanishes on each face as well, so $v \in \mathcal{P}_{k+3}^{0}\left(K ; \mathbb{R}^{3}\right)$. This completes the proof of the second statement.

The third statement is an immediate consequence of the first two and the fact that $\mathbb{T} \cap \mathcal{P}_{k+3}^{0}\left(K ; \mathbb{R}^{3}\right)=0$.

Theorem 7.2. For $k \geq 4$ the space $\mathcal{M}_{k}(K)$ has dimension $(k+2)(k-2)(k-3) / 2$.

Proof. Using first the short exact sequence in the lemma and then the dimension formula in Theorem 6.6] we get

$$
\begin{aligned}
\operatorname{dim} \mathcal{M}_{k} & =\operatorname{dim} \mathcal{N}_{k+2}-\operatorname{dim} \epsilon\left[\mathcal{P}_{k+3}^{0}\left(K ; \mathbb{R}^{3}\right)\right] \\
& =(k+2)\left(k^{2}-2 k+3\right)-\operatorname{dim} \mathcal{P}_{k-1}\left(K ; \mathbb{R}^{3}\right)=(k+2)(k-2)(k-3) / 2 .
\end{aligned}
$$


To conclude, we construct a basis for the space $\mathcal{M}_{k}(K)$ for $k=4$ and $k=5$. (Alternatively a basis could be constructed for any $k$ using computational algebra software.) For this we use the following lemma, similar to Lemma 7.1.

Lemma 7.3. (1) The operator curl curl ${ }^{*}$ maps $b_{K} \mathcal{N}_{k-2}^{0}(K)$ onto $\mathcal{M}_{k}(K)$.

(2) $\left\{T \in b_{K} \mathcal{N}_{k-2}^{0} \mid \operatorname{curl}^{\operatorname{curl}^{*}} T=0\right\}=\epsilon\left[b_{K}^{2} \mathcal{P}_{k-5}\left(K ; \mathbb{R}^{3}\right)\right]$.

(3) The following sequence is exact:

$$
0 \rightarrow b_{K}^{2} \mathcal{P}_{k-5}\left(K ; \mathbb{R}^{3}\right) \stackrel{\epsilon}{\rightarrow} b_{K} \mathcal{N}_{k-2}^{0}(K) \stackrel{\text { curl curl }^{*}}{\longrightarrow} \mathcal{M}_{k}(K) \rightarrow 0 .
$$

Proof. Note that $b_{K} \mathcal{N}_{k-2}^{0} \subset \mathcal{N}_{k+2}$ by (6.13), and so curl curl ${ }^{*} b_{K} \mathcal{N}_{k-2}^{0} \subset \mathcal{M}_{k}(K)$.

First we prove (2). Let $w \in \mathcal{P}_{k-5}\left(K ; \mathbb{R}^{3}\right)$. By the Leibniz rule,

$$
\epsilon\left(b_{K}^{2} w\right)=b_{K}^{2} \epsilon(w)+b_{K}\left[\left(\operatorname{grad} b_{K}\right) w^{\prime}+w\left(\operatorname{grad} b_{K}\right)^{\prime}\right] .
$$

Clearly $b_{K} \epsilon(w) \in \mathcal{N}_{k-2}^{0}$, and, recalling that $\operatorname{grad} b_{K}=-b_{f} n_{f} / h_{f}$, we see that $\left(\operatorname{grad} b_{K}\right) w^{\prime}+w\left(\operatorname{grad} b_{K}\right)^{\prime} \in \mathcal{N}_{k-2}^{0}$. Thus $\epsilon\left(b_{K}^{2} w\right) \in b_{K} \mathcal{N}_{k-2}^{0}$, giving the inclusion $\supset$. Conversely, if $T \in b_{k} \mathcal{N}_{k-2}^{0}$ with curl curl ${ }^{*} T=0$, then, by Lemma 7.1] $T=\epsilon\left(b_{K} v\right)$ for some $v \in \mathcal{P}_{k-1}\left(K ; \mathbb{R}^{3}\right)$ and we need to show that $v=0$ on $\partial K$. Using the Leibniz rule and the fact that $T$ vanishes on $\partial K$, we get that $n v^{\prime}+v n^{\prime}$ vanishes on each face. We conclude that $v$ vanishes on the face, using the elementary identity $v=\left(I+Q_{n}\right)\left(n v^{\prime}+v n^{\prime}\right) n / 2$.

It follows that

$$
\operatorname{dim}\left[\operatorname{curl} \operatorname{curl}^{*}\left(b_{K} \mathcal{N}_{k-2}^{0}\right)\right]=\operatorname{dim} \mathcal{N}_{k-2}^{0}-\operatorname{dim} \mathcal{P}_{k-5}\left(K ; \mathbb{R}^{3}\right)=\operatorname{dim} \mathcal{M}_{k},
$$

where we have used Lemma 6.1 and Theorem 7.2 , The exactness of (7.6), and so also the first statement of the lemma, follows.

Thus for $k=4$, curlcurl ${ }^{*}$ is injective on $b_{K} \mathcal{N}_{2}^{0}$, and so a basis for $\mathcal{M}_{4}=$ $\operatorname{curl} \operatorname{curl}^{*}\left(b_{K} \mathcal{N}_{2}^{0}\right)$ is computable directly from a basis for $\mathcal{N}_{2}^{0}$, which may be obtained directly from (6.3).

Now let $k=5$. The map curl curl ${ }^{*}$ is not injective on $b_{K} \mathcal{N}_{3}^{0}$, but has a kernel of dimension 3. In this case, the representation (6.3) presents an arbitrary element $S \in \mathcal{N}_{3}^{0}$ as

$$
S=\sum_{e \in \Delta_{1}(K)} b_{e} S_{e}+\sum_{f \in \Delta_{2}(K)} b_{f} S_{f}, \quad S_{e} \in \mathcal{P}_{1}^{e}\left(K ; N^{e}\right), S_{f} \in N^{f} .
$$

Fix a particular face $f_{0} \in \Delta_{2}(K)$ and define $\mathcal{N}_{3}^{00}$ as the subspace of $S \in \mathcal{N}_{3}^{0}$ for which $S_{f_{0}}=0$ in this representation, clearly a subspace of codimension 3 . We claim that curl curl ${ }^{*}$ is injective on the space $b_{K} \mathcal{N}_{3}^{00}$, and hence a basis for $\mathcal{M}_{5}$ can be computed from a corresponding basis of $\mathcal{N}_{3}^{00}$. The injectivity follows since if $w \in \mathbb{R}^{3}$, with $\epsilon\left(b_{K}^{2} w\right) \in b_{K} \mathcal{N}_{3}^{00}$, then we get $w=0$ arguing as in the proof of Lemma 7.3

\section{ACKNOWLEDGEMENT}

The third author is grateful to Snorre Christiansen for many useful discussions. 


\section{REFERENCES}

1. Scot Adams and Bernardo Cockburn, A mixed finite element method for elasticity in three dimensions, J. Sci. Comput. 25 (2005), no. 3, 515-521. MR2221175(2006m:65251)

2. M. Amara and J. M. Thomas, Equilibrium finite elements for the linear elastic problem, Numer. Math. 33 (1979), no. 4, 367-383. MR 553347 (81b:65096)

3. Douglas N. Arnold, Differential complexes and numerical stability, Proceedings of the International Congress of Mathematicians, Vol. I (Beijing, 2002) (Beijing), Higher Ed. Press, 2002, pp. 137-157. MR.1989182(2004h:65115)

4. Douglas N. Arnold and Gerard Awanou, Rectangular mixed finite elements for elasticity, Math. Models Methods Appl. Sci. 15 (2005), no. 9, 1417-1429. MR2166210 (2006f:65112)

5. Douglas N. Arnold, Franco Brezzi, and Jim Douglas, Jr., PEERS: a new mixed finite element for plane elasticity, Japan J. Appl. Math. 1 (1984), no. 2, 347-367. MR840802 (87h:65189)

6. Douglas N. Arnold, Jim Douglas, Jr., and Chaitan P. Gupta, A family of higher order mixed finite element methods for plane elasticity, Numer. Math. 45 (1984), no. 1, 1-22. MR761879 (86a:65112)

7. Douglas N. Arnold and Richard S. Falk, A new mixed formulation for elasticity, Numer. Math. 53 (1988), no. 1-2, 13-30. MR946367 (89f:73020)

8. Douglas N. Arnold, Richard S. Falk, and Ragnar Winther, Mixed finite element methods for linear elasticity with weakly imposed symmetry, Math. Comp. 76 (2007), 1699-1723. MR2336264

9. - Finite element exterior calculus, homological techniques, and applications, Acta Numer. 15 (2006), 1-155. MR2269741

10. Douglas N. Arnold and Ragnar Winther, Mixed finite elements for elasticity, Numer. Math. 92 (2002), no. 3, 401-419. MR1930384 (2003i:65103)

11. F. Brezzi, On the existence, uniqueness and approximation of saddle-point problems arising from Lagrangian multipliers, Rev. Française Automat. Informat. Recherche Opérationnelle Sér. Rouge 8 (1974), no. R-2, 129-151. MR0365287 (51:1540)

12. Franco Brezzi and Michel Fortin, Mixed and hybrid finite element methods, Springer Series in Computational Mathematics, vol. 15, Springer-Verlag, New York, 1991. MR 1115205 (92d:65187)

13. Ph. Clément, Approximation by finite element functions using local regularization, Rev. Française Automat. Informat. Recherche Opérationnelle Sér., RAIRO Analyse Numérique 9 (1975), no. R-2, 77-84. MR0400739 (53:4569)

14. R. S. Falk and J. E. Osborn, Error estimates for mixed methods, RAIRO Anal. Numér. 14 (1980), no. 3, 249-277. MR592753 (82j:65076)

15. Badouin M. Fraejis de Veubeke, Displacement and equilibrium models in the finite element method, Stress analysis (New York) (O.C Zienkiewics and G.S. Holister, eds.), Wiley, 1965, pp. $145-197$.

16. C. Johnson and B. Mercier, Some equilibrium finite element methods for two-dimensional elasticity problems, Numer. Math. 30 (1978), no. 1, 103-116. MR0483904 (58:3856)

17. E. Stein and R. Rolfes, Mechanical conditions for stability and optimal convergence of mixed finite elements for linear plane elasticity, Comput. Methods Appl. Mech. Engrg. 84 (1990), no. 1, 77-95. MR1082821(91i:73045)

18. R. Stenberg, On the construction of optimal mixed finite element methods for the linear elasticity problem, Numer. Math. 48 (1986), no. 4, 447-462. MR834332 (87i:73062)

19. - A family of mixed finite elements for the elasticity problem, Numer. Math. 53 (1988), no. 5, 513-538. MR 954768(89h:65192)

20. - Two low-order mixed methods for the elasticity problem, The mathematics of finite elements and applications, VI (Uxbridge, 1987), Academic Press, London, 1988, pp. 271-280. MR $956898(89 \mathrm{j}: 73074)$

21. V.B. Watwood Jr. and B.J. Hartz, An equilibrium stress field model for finite element solution of two-dimensional elastostatic problems, Internat. J. Solids Structures 4 (1968), 857-873. 
Institute for Mathematics and its Applications, University of Minnesota, MinneapoLis, Minnesota 55455

E-mail address: arnold@ima.umn.edu

Department of Mathematical Sciences, Northern Illinois University, Dekalb, IlliNOIS 60115

E-mail address: awanou@math.niu.edu

Centre of Mathematics for Applications and Department of Informatics, University of Oslo, P.O. Box 1053, Blindern, 0316 Oslo, Norway

E-mail address: ragnar.winther@cma.uio.no 